Interfaces and Free Boundaries 13 (2011), 1-27

DOI $10.4171 / \mathrm{IFB} / 246$

\title{
A generalized Cahn-Hilliard equation incorporating geometrically linear elasticity
}

\author{
THOMAS BLESGEN \\ Max-Planck-Institute for Mathematics in the Sciences, \\ Inselstraße 22-26, 04103 Leipzig, Germany \\ E-mail: blesgen@mis.mpg.de \\ ISAAC V. CHENCHIAH \\ Department of Mathematics, University of Bristol, \\ University Walk, Bristol BS8 1TW, UK \\ E-mail: Isaac.Chenchiah@bristol.ac.uk
}

[Received 4 December 2008 and in revised form 23 July 2010]

\begin{abstract}
We consider a generalisation of the Cahn-Hilliard equation that incorporates an elastic energy density which, being quasiconvex, incorporates microstructure formation on smaller length scales. We prove global existence of weak solutions in certain microstructural regimes in (one and) two dimensions and present sufficient conditions for uniqueness. Preliminary numerical computations to illustrate some characteristic properties of the solutions are presented and compared to earlier Cahn-Hilliard models with elasticity.
\end{abstract}

2010 Mathematics Subject Classification: Primary 35K55, 74B99, 74Q15; Secondary 35K40, 65M60, 74N99.

Keywords: Cahn-Hilliard equation; coarsening; elasticity.

\section{Introduction}

The dependence of macroscopic properties on microstructure is considerably involved in situations where the microstructure itself can change with deformation, as, e.g., in solids that undergo phase transformations. The modeling of such solids at macroscopic length scales involves characterising the microstructures that form in them and their change as a result of macroscopic deformation.

Two such situations are of particular importance: The first is the shape memory effect which is the temperature-induced recovery of apparently plastic deformation. This phenomenon is the result of martensitic phase transformation, [8, 9]. The second occurs in superalloys, [55], which are of great importance to turbine blades. These alloys are precipitate hardened: An alloy with offstoichiometric composition is quenched to create numerous precipitates which then increase the creep resistance of the alloy. Here the key problem is to understand the equilibrium morphology of the precipitates and its dependence on external loads.

In the present article we shall address this problem by combining the Cahn-Hilliard model with recent results in [18] where the relaxation of two-well elastic energies with given volume fraction is studied. We restrict ourselves to the (one- and) two-dimensional case, as only partial relaxation results are available in three dimensions. Moreover lengthy explicit estimates of the energy are needed that differ in two and three dimensions. 
Outline. We derive the new Cahn-Hilliard model in Section 2 and address existence and uniqueness of weak solutions in Section 3. The next section is devoted to numerics: three case studies in Section 4.2 reveal some of the properties of the new model. We end with some closing remarks. Ancillary calculations are gathered in the appendices.

Notation. Adopting common notation, - denotes the inner product in $\mathbb{R}^{D}$, and : the inner product in $\mathbb{R}^{D \times D}$, i.e., for $A, B \in \mathbb{R}^{D \times D}, A: B:=\operatorname{tr}\left(A^{T} B\right)=\sum_{i, j=1}^{D} A_{i j} B_{i j}$.

\section{The Cahn-Hilliard equation with elasticity}

\subsection{The elastic Cahn-Hilliard equation}

The Cahn-Hilliard equation is a particular example of a Landau-Ginzburg equation and was introduced in [14]. It describes the segregation of a two-phase solid under isothermal conditions for a temperature $\theta$ below the critical temperature $\theta_{c}$ of the material. In contrast to, e.g., the AllenCahn equation, the order parameter is a conserved quantity, i.e., its integral over the whole domain does not change with time.

Substantial progress has been made in the theoretical understanding of the Cahn-Hilliard model, as evidenced by the generalisation to multiple phases [25], to non-isothermal settings [3, 4], to concentration-dependent mobilities [13]; incorporation of convective [57] and viscous [42, 49] effects; coupling to the Navier-Stokes equations [10, 36]; the investigation of many limiting cases [2, 17, 22]; the existence of general Cahn-Hilliard/Allen-Cahn models [16]; and the extension of the model to related problems in materials science [11, 34]. The cited articles are only a small selection and there exist many other significant works.

Our focus here is on the elastic Cahn-Hilliard equation which is the Cahn-Hilliard equation extended to incorporate elastic effects [35, 31, 32, 33]. Its general form is

$$
\begin{aligned}
\partial_{t} \chi & =\operatorname{div}(L \nabla \mu), \\
\mu & =-\gamma \Delta \chi+\psi^{\prime}(\chi)+\partial_{\chi} \widehat{W}(\chi, \varepsilon(\mathbf{u})), \\
0 & =\operatorname{div}\left(\partial_{\varepsilon} \widehat{W}(\chi, \varepsilon(\mathbf{u}))\right)
\end{aligned}
$$

to be solved in a space-time cylinder $\Omega_{S}:=\Omega \times(0, S)$, where $S>0$ denotes a stop time and $\Omega \subset$ $\mathbb{R}^{D}$ is a bounded domain with Lipschitz boundary containing the solid. Here $\chi: \Omega_{S} \rightarrow[0,1]$ is the order parameter which coincides with the mass fraction of one phase of the solid. For convenience we also define

$$
\chi_{1}:=\chi, \quad \chi_{2}:=(1-\chi) .
$$

Equation 2.1a) expresses conservation of mass: $\partial_{t} \chi=-\operatorname{div} J$, where the flux $J$ is related to the chemical potential $\mu$ through Onsager's relation [51, 52], $J=-L \nabla \mu$. (We remark that this relation is phenomenological and may be invalid when the system is far from thermodynamic equilibrium.)

Equation 2.1b states that the chemical potential is the derivative of the free-energy density

$$
F(\chi, \mathbf{u}):=\int_{\Omega}\left(\psi(\chi)+\frac{\gamma}{2}|\nabla \chi|^{2}+\widehat{W}(\chi, \varepsilon(\mathbf{u}))-\bar{\sigma}: \varepsilon(\mathbf{u})\right) \mathrm{d} x .
$$

The entropic part of the free energy is

$$
\psi(\chi)=k_{B} \theta(\chi \ln (\chi)+(1-\chi) \ln (1-\chi))+\frac{\theta_{c}}{2} \chi(1-\chi)
$$


note that this is a double-well potential. The interfacial part of the free energy, $\frac{\gamma}{2}|\nabla \chi|^{2}$, influences the thickness of the interfacial layer; it neglects anisotropy and surface diffusion, see e.g., [19, 5, 7] for generalisations. The elastic part of the free energy, $\widehat{W}(\chi, \varepsilon)$, is defined in Section 2.2. for now we note that it is a function of the strain $\varepsilon$ which depends on the displacement $\mathbf{u}: \Omega_{S} \rightarrow \mathbb{R}^{D}$ through

$$
\varepsilon(\mathbf{u}):=\frac{1}{2}\left(\nabla \mathbf{u}+\nabla \mathbf{u}^{T}\right) .
$$

The last term is the contribution to the free energy density due to tractions at the boundary.

The Cauchy stress in the system is given by $\sigma:=\partial_{\varepsilon} \widehat{W}(\chi, \varepsilon(\mathbf{u}))$ and thus $\left.2.1 \mathrm{c}\right)$ is a consequence of Newton's second law under the assumption that the acceleration $\partial_{t t} \mathbf{u}$ is negligible. The CahnHilliard equation with non-stationary elasticity has been studied in [54].

Boundary conditions. The system 2.1) is solved with the boundary conditions on $\partial \Omega$,

$$
\begin{gathered}
L \nabla \mu \cdot \mathbf{n}=0 \\
\gamma \nabla \chi \cdot \mathbf{n}=0 \\
\mathbf{u}=\bar{\varepsilon} x \quad \text { or } \quad \partial_{\varepsilon} \widehat{W}(\chi, \varepsilon(\mathbf{u})) \mathbf{n}=\bar{\sigma} \mathbf{n},
\end{gathered}
$$

where $\mathbf{n}$ is the unit outer normal to $\partial \Omega$ and $\bar{\varepsilon}, \bar{\sigma} \in \mathbb{R}_{\text {sym }}^{D \times D}$. The first of these enforces zero mass flux across the boundary and the last imposes either displacement or traction $\mathbf{t}=\bar{\sigma} \mathbf{n}$ at the boundary. The condition $\gamma \nabla \chi \cdot \mathbf{n}=0$ is not physically motivated. It is chosen here to guarantee the uniqueness of the mathematical solution in certain cases.

In the absence of body forces, the work necessary to transform the undeformed body $\Omega$ into a state with displacement $\mathbf{u}$ is

$$
\begin{aligned}
-\int_{\partial \Omega} \mathbf{u} \cdot \mathbf{t} & =-\int_{\partial \Omega} \sum_{k=1}^{D} \mathbf{u}_{k} \sum_{j=1}^{D} \bar{\sigma}_{k j} \mathbf{n}_{j}=-\int_{\partial \Omega} \sum_{j, k=1}^{D} \bar{\sigma}_{j k} \mathbf{u}_{k} \mathbf{n}_{j} \\
& =-\int_{\Omega} \operatorname{div}(\bar{\sigma} \mathbf{u})=-\int_{\Omega} \nabla \mathbf{u}: \bar{\sigma}=-\int_{\Omega} \varepsilon(\mathbf{u}): \bar{\sigma}
\end{aligned}
$$

where we use that $\bar{\sigma}$ is symmetric and constant. This explains the last integrand in 2.3 .

The second law of thermodynamics. We end this section by a short verification of the second law of thermodynamics for the system (2.1), 2.6. A direct computation shows

$$
\begin{aligned}
\frac{\mathrm{d}}{\mathrm{d} t} F & (\chi(t), \mathbf{u}(t)) \\
& =\int_{\Omega}\left(\gamma \nabla \chi \cdot \nabla \partial_{t} \chi+\psi^{\prime}(\chi) \partial_{t} \chi+\partial_{\chi} \widehat{W}(\chi, \varepsilon(\mathbf{u})) \partial_{t} \chi+\sigma: \partial_{t} \varepsilon(\mathbf{u})-\bar{\sigma}: \partial_{t} \varepsilon(\mathbf{u})\right) \mathrm{d} x \\
& =\int_{\Omega}\left(-\gamma \Delta \chi+\psi^{\prime}(\chi)+\partial_{\chi} \widehat{W}(\chi, \varepsilon)\right) \partial_{t} \chi \mathrm{d} x+\int_{\partial \Omega}\left(\gamma \partial_{t} \chi \nabla \chi \cdot \mathbf{n}+(\sigma \mathbf{n}-\mathbf{t}) \cdot \partial_{t} \mathbf{u}\right) \mathrm{d} x \\
& =\int_{\Omega} \mu \operatorname{div}(L \nabla \mu) \mathrm{d} x+\int_{\partial \Omega}\left(\gamma \partial_{t} \chi \nabla \chi \cdot \mathbf{n}+(\sigma \mathbf{n}-\mathbf{t}) \cdot \partial_{t} \mathbf{u}\right) \mathrm{d} x \\
& =-\int_{\Omega} L \nabla \mu \cdot \nabla \mu \mathrm{d} x+\int_{\partial \Omega}\left(\mu L \nabla \mu \cdot \mathbf{n}+\gamma \partial_{t} \chi \nabla \chi \cdot \mathbf{n}+(\sigma \mathbf{n}-\mathbf{t}) \cdot \partial_{t} \mathbf{u}\right) \mathrm{d} x
\end{aligned}
$$


where we employed 2.6) and $\sigma: \partial_{t} \varepsilon(\mathbf{u})=\operatorname{div}\left(\sigma \partial_{t} \mathbf{u}\right)$, which holds as $\sigma, \bar{\sigma}$ are symmetric, $\bar{\sigma}$ is constant by assumption, and because of $2.1 \mathrm{c}$. .

As $L$ is positive semi-definite we have $L \nabla \mu \cdot \nabla \mu \geqslant 0$; this is the entropy production due to diffusion. The term $\int_{\partial \Omega} \mu L \nabla \mu \cdot \mathbf{n}$ describes energy inflow due to diffusion and $\int_{\partial \Omega} \gamma \partial_{t} \chi \nabla \chi \cdot \mathbf{n}$ that due to moving interfaces. Finally, $\int_{\partial \Omega}(\sigma \mathbf{n}-\mathbf{t}) \cdot \partial_{t} \mathbf{u}$ is the power expended due to the deformation stresses. For a thermodynamically closed system with boundary conditions 2.6 , the boundary integrals vanish and the above equality shows that the second law of thermodynamics is fulfilled.

\subsection{The elastic energy density}

In previous work, e.g. [15, 35, 32, 53], the Cahn-Hilliard system has been investigated under the assumption, dating back to Eshelby [26], that the elastic energy density is of the form

$$
\begin{aligned}
W_{\operatorname{lin}}(\chi, \varepsilon) & :=\frac{1}{2} \alpha\left(\varepsilon-\varepsilon^{T}(\chi)\right):\left(\varepsilon-\varepsilon^{T}(\chi)\right), \\
\varepsilon^{T}(\chi) & :=\chi_{1} \varepsilon_{1}^{T}+\chi_{2} \varepsilon_{2}^{T} .
\end{aligned}
$$

We refer to this as the 'linear model'. Note that $\alpha$, the elastic modulus, is assumed to be the same for both phases and, critically, $\varepsilon^{T}(\chi)$ is a linear interpolation between the stress-free strains $\varepsilon_{1}^{T}, \varepsilon_{2}^{T}$ of the two phases. The resulting stress-strain relationship for the mixture is linear: this is Vegard's law.

Vegard's law has been observed to fail in situations of great technological interest where the microstructure is known to be decisive for macroscopic properties, like, e.g., for Ga/As precipitates [56] and $\mathrm{Ge} / \mathrm{Si}$ heterostructures [47]. An overview when Vegard's law fails is attempted in [28].

Elastic energy densities through relaxation. We propose here to replace $W_{\text {lin }}$ by a rigorouslyderived elastic energy density. We begin, like our predecessors, with two linearly-elastic phases:

$$
W_{i}(\varepsilon):=\frac{1}{2} \alpha_{i}\left(\varepsilon-\varepsilon_{i}^{T}\right):\left(\varepsilon-\varepsilon_{i}^{T}\right)+w_{i}, \quad i=1,2,
$$

except that we do not require the elastic moduli $\alpha_{i}$ to be equal. (By $\varepsilon_{i}^{T}$, we denote the stress-free strain of phase $i$ and the constants $w_{i} \in \mathbb{R}$ allow for the possibility that the two phases have unequal energy minima.) However, instead of postulating a form (linear or otherwise) for the energy density of a mixture of the two phases, we derive it rigorously. Our reasoning is as follows:

We interpret the order parameter $\chi(x) \in[0,1]$ as prescribing the volume fractions of the two phases at $x \in \Omega$, i.e., in a ball $B_{r}(x) \subset \Omega$. (It is tacitly assumed that the regions occupied by each of the two phases in $B_{r}(x)$ are measurable.) Thus, if $\tilde{\chi}_{1} \equiv \tilde{\chi}, \tilde{\chi}_{2}=1-\tilde{\chi}$ are the characteristic functions of the two phases on the microscale, we have $\tilde{\chi}_{i} \in B V\left(B_{r}(x) ;\{0,1\}\right)$ and

$$
\chi_{i}(x)=\left\langle\tilde{\chi}_{i}\right\rangle:=\frac{1}{\left|B_{r}(x)\right|} \int_{B_{r}(x)} \tilde{\chi}_{i}(y) \mathrm{d} y, \quad i=1,2,
$$

where $|E|$ is the $D$-dimensional Lebesgue measure of a set $E$. In the absence of microstructural surface energy, the elastic energy of this ball for a microscale deformation $\tilde{\mathbf{u}}$ is

$$
\int_{B_{r}(x)}\left(\tilde{\chi}_{1} W_{1}(\varepsilon(\tilde{\mathbf{u}}))+\tilde{\chi}_{2} W_{2}(\varepsilon(\tilde{\mathbf{u}}))\right) \mathrm{d} y .
$$


Next we postulate that the system selects $\tilde{\chi}$ and $\tilde{\mathbf{u}}$ to minimise the microscopic elastic energy. (In our context this implies, in particular, that the deformations adopt instantaneously to diffusion-induced changes in concentration.) This reasoning leads us to conclude that the macroscopic elastic energy density is

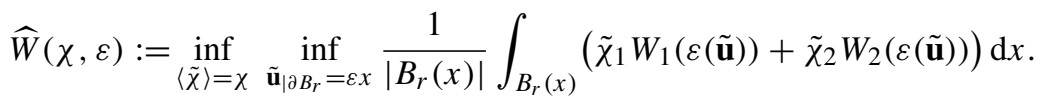

This definition of $\widehat{W}$ is in fact independent of $r$ (see, e.g., [21]). Mathematically, the infimum over $\tilde{\chi}$ in $(2.9)$ is the result of relaxation subject to prescribed volume fractions (cf. [41]).

$\widehat{W}$ in two dimensions. The (analytical) computation of $\widehat{W}$ is non-trivial for dimensions larger than one. For two dimensions, an explicit (albeit involved) expression was derived in [18]. We reproduce it here; this requires first defining some intermediate quantities.

With $T: \mathbb{R}_{\mathrm{sym}}^{2 \times 2} \rightarrow \mathbb{R}_{\mathrm{sym}}^{2 \times 2}$ being the linear operator defined by

$$
T \varepsilon:=\varepsilon-\operatorname{tr}(\varepsilon) \operatorname{Id},
$$

let $\gamma_{i}>0$ be the reciprocal of the largest eigenvalue of $\alpha_{i}^{-1 / 2} T \alpha_{i}^{-1 / 2}$ and

$$
\gamma^{*}:=\min \left\{\gamma_{1}, \gamma_{2}\right\}
$$

Next, for $\beta \in\left[0, \gamma^{\star}\right]$, let

$$
\begin{aligned}
\alpha(\beta, \chi) & :=\chi_{2} \alpha_{1}+\chi_{1} \alpha_{2}-\beta T, \\
\Delta \varepsilon^{*}(\beta, \chi, \varepsilon) & :=\alpha^{-1}\left(\beta^{*}, \chi\right)\left(\left(\alpha_{1}-\alpha_{2}\right) \varepsilon+\left(\alpha_{2} \varepsilon_{2}^{T}-\alpha_{1} \varepsilon_{1}^{T}\right)\right), \\
\varphi(\beta, \chi, \varepsilon) & :=-\operatorname{det} \Delta \varepsilon^{*}(\beta, \chi, \varepsilon),
\end{aligned}
$$

and let

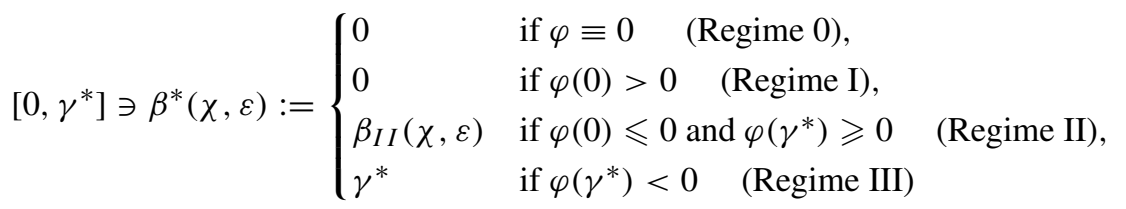

where $\beta_{I I}(\chi, \varepsilon)$ is the unique solution of $\varphi(\cdot, \chi, \varepsilon)=0$. Finally let

$$
\begin{aligned}
& \varepsilon_{1}^{*}\left(\beta^{*}, \chi, \varepsilon\right):=\alpha^{-1}\left(\beta^{*}, \chi\right)\left(\left(\alpha_{2}-\beta^{*} T\right) \varepsilon-\chi_{2}\left(\alpha_{2} \varepsilon_{2}^{T}-\alpha_{1} \varepsilon_{1}^{T}\right)\right), \\
& \varepsilon_{2}^{*}\left(\beta^{*}, \chi, \varepsilon\right):=\alpha^{-1}\left(\beta^{*}, \chi\right)\left(\left(\alpha_{1}-\beta^{*} T\right) \varepsilon+\chi_{1}\left(\alpha_{2} \varepsilon_{2}^{T}-\alpha_{1} \varepsilon_{1}^{T}\right)\right), \\
& \Delta \varepsilon^{*}(\beta, \chi, \varepsilon)=\varepsilon_{2}^{*}(\beta, \chi, \varepsilon)-\varepsilon_{1}^{*}(\beta, \chi, \varepsilon) .
\end{aligned}
$$

Then, in two dimensions,

$$
\widehat{W}(\chi, \varepsilon)=\chi_{1} W_{1}\left(\varepsilon_{1}^{*}\right)+\chi_{2} W_{2}\left(\varepsilon_{2}^{*}\right)+\beta^{*} \chi_{1} \chi_{2} \operatorname{det}\left(\varepsilon_{2}^{*}-\varepsilon_{1}^{*}\right) .
$$

It can be shown that this is well defined and the four regimes are mutually exclusive. The different regimes are associated with different microstructures: 
0 . The phases are elastically indistinguishable in that the energy does not depend on the microstructure. This occurs when $\alpha_{1}\left(\varepsilon-\varepsilon_{1}^{T}\right)=\alpha_{2}\left(\varepsilon-\varepsilon_{2}^{T}\right)$.

I. There exist two optimal rank-one laminates.

II. The unique optimal microstructure is a rank-one laminate.

III. There exist two optimal rank-two laminates, other microstructures can also exist.

Figure 1 illustrates rank-one and rank-two laminates.
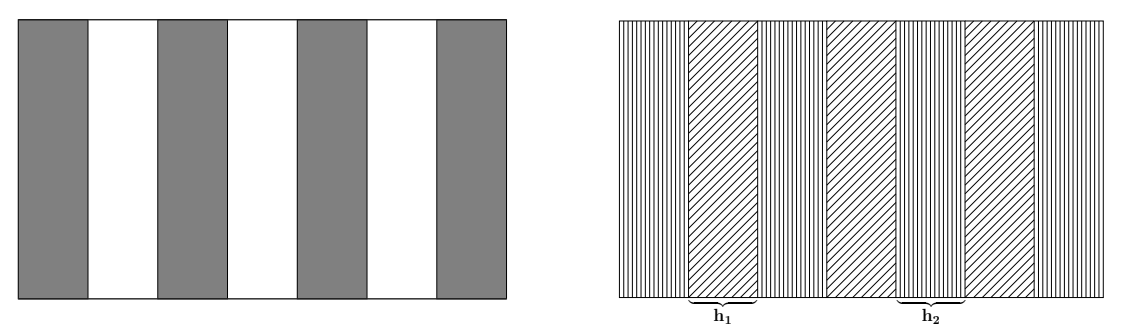

FIG. 1. Two-phase rank-one and rank-two laminates in two-dimensions. The strain is (globally) constant in each shaded region. Left: A rank-one laminate. In this illustration the volume fraction of each phase is 0.5. Right: A rank-two laminate. Note the separation in length-scale between the layers.

REMARK 2.1 The linear model can be recovered as a special case of the geometrically linear theory. In two space-dimensions, sufficient conditions are $\alpha_{1}=\alpha_{2}$ and

$$
\operatorname{det}\left(\varepsilon_{2}^{T}-\varepsilon_{1}^{T}\right) \leqslant 0
$$

Indeed, for $\alpha_{1}=\alpha_{2}$, the mapping $\varphi=\varphi(\beta)$ is independent of $\chi, \varepsilon$. If the inequality in 2.11 is strict, we are in Regime I, otherwise in Regime 0 ; in both cases $\beta^{*} \equiv 0$ in $\Omega_{S}$ and

$$
\varepsilon_{1}^{*}=\varepsilon_{1}^{*}(\chi, \varepsilon)=\varepsilon-\chi_{2}\left(\varepsilon_{2}^{T}-\varepsilon_{1}^{T}\right), \quad \varepsilon_{2}^{*}=\varepsilon_{2}^{*}(\chi, \varepsilon)=\varepsilon+\chi_{1}\left(\varepsilon_{2}^{T}-\varepsilon_{1}^{T}\right)
$$

such that

In combination with $(2.10 \mathrm{~h}$ ) we find

$$
\varepsilon_{1}^{*}-\varepsilon_{1}^{T}=\varepsilon-\chi_{1} \varepsilon_{1}^{T}-\chi_{2} \varepsilon_{2}^{T}=\varepsilon_{2}^{*}-\varepsilon_{2}^{T} .
$$

$$
\widehat{W}(\chi, \varepsilon)=\frac{1}{2}\left(\alpha_{1}\left(\varepsilon-\chi_{1} \varepsilon_{1}^{T}-\chi_{2} \varepsilon_{2}^{T}\right)\right):\left(\varepsilon-\chi_{1} \varepsilon_{1}^{T}-\chi_{2} \varepsilon_{2}^{T}\right)+\chi_{1} w_{1}+\chi_{2} w_{2}
$$

showing

$$
\begin{aligned}
\operatorname{div}\left(\partial_{\varepsilon} \widehat{W}(\chi, \varepsilon)\right) & =\operatorname{div}\left(\alpha_{1}\left(\varepsilon-\chi_{1} \varepsilon_{1}^{T}-\chi_{2} \varepsilon_{2}^{T}\right)\right)=\operatorname{div}\left(\alpha_{1}\left(\varepsilon-\left(\varepsilon_{1}^{T}-\varepsilon_{2}^{T}\right) \chi\right)\right) \\
& =\operatorname{div}\left(\partial_{\varepsilon} \widehat{W}_{\text {lin }}(\chi, \varepsilon)\right),
\end{aligned}
$$

using (2.7b). For the case $D=3$, we refer the reader to [18, Note 4.30].

REMARK 2.2 Explicit expressions for $\widehat{W}$ in one dimension are found in [12]: $\beta^{*}=0$ and

$$
\begin{aligned}
\widehat{W}(\chi, \varepsilon) & =\chi W_{1}\left(\varepsilon_{1}^{*}\right)+(1-\chi) W_{2}\left(\varepsilon_{2}^{*}\right), \\
\varepsilon_{1}^{*}(\chi, \varepsilon) & =\frac{\alpha_{2}\left(\varepsilon-\chi_{2} \varepsilon_{2}^{T}\right)+\chi_{2} \alpha_{1} \varepsilon_{1}^{T}}{\chi_{2} \alpha_{1}+\chi_{1} \alpha_{2}}, \\
\varepsilon_{2}^{*}\left(\chi_{1}, \varepsilon\right) & =\frac{\alpha_{1}\left(\varepsilon-\chi_{1} \varepsilon_{1}^{T}\right)+\chi_{1} \alpha_{2} \varepsilon_{2}^{T}}{\chi_{2} \alpha_{1}+\chi_{1} \alpha_{2}}
\end{aligned}
$$


with $\alpha_{i}, \varepsilon_{i}^{*} \in \mathbb{R}$. Partial results are available for three dimensions [18]. Explicit expressions are unknown (although arguably not necessary) for dimensions larger than three.

\subsection{Comparison with other approaches}

Existing models for segregation and phase-change phenomena in the framework of elasticity roughly fall in one of three categories.

Atomistic models constitute the first category. On a micromechanical level atomistic models are used to simulate the behaviour of atoms inside the lattice structure, [38]. These models are based on probability assumptions describing the exchange of atoms on the lattice (with, e.g., Kawasaki dynamics). The downside of these models is that it is only possible to simulate a limited amount of atoms which in general is not sufficient for realistic simulations. In addition, no analytic formulae for the elastic energy can be derived from these computations.

The second category originates from concentration-dependent models with sharp interfaces. For example, in [37, 43], the diffusional evolution of microstructure in binary alloys is studied based on a sharp interface model and a general Gibbs-Thomson law. Inside the bulk phase, Fick's law of diffusion with constant diffusivity is assumed and the numerical solver is accelerated by a preconditioner taylor-made for the Laplace operator. A related sharp interface model has been studied numerically in [1].

The last category is formed by concentration-dependent models with diffuse interfaces. Motivated by experimental observations of coherent inclusions in elastic media, a simple theoretical model for microstructure evolution in the absence of diffusion is developed in [40, 44]. Gurtin [36] considers a coupling of the Cahn-Hilliard equation with the force balance (2.1c) and the microforce balance $\operatorname{div} \xi+\pi+\gamma=0$, where $\xi$ are microstresses, $\pi$ internal microforces and $\gamma$ external microforces. The existence and uniqueness of weak solutions to this model is treated in [45, 46]. In contrast to this microforce balance, our model is based on local elastic energy minimisation. In [29, 30] the ansatz is supplemented by thermodynamically consistent constitutive relations. The microforce balance then yields a generalisation of the elastic Cahn-Hilliard model where the chemical potential is derived as variational derivative of the total free energy with respect to atomic densities. The integration of the configurational force balance over the transition layer yields in the limit the generalised Gibbs-Thomson law of the sharp-interface theory.

The articles [23, 24] pursue a more engineering approach to simulate coarsening in tin/lead solders. The authors start from an incoherent structure and study the local stresses and strains numerically in representative volume elements. In contrast to [29, 36], the analytical model is solely based on a continuum description. By the Cahn-Hilliard equation, surface tension is incorporated. The model uses linearised kinematics and for computing the effective elastic modulus in the interfacial region, a linear interpolation ansatz between the (constant) elastic moduli $\alpha_{1}, \alpha_{2}$ of the two phases is made.

\section{Existence and uniqueness of weak solutions}

\subsection{Assumptions}

The proof of an earlier existence result for the Cahn-Hilliard equation with linear elasticity and either polynomial [31, 32] or logarithmic [31, 33] free energy can be adopted to prove existence of weak solutions to the system (2.1), 2.4], (2.10) provided the nonlinear elastic energy $\widehat{W}$ satisfies the following assumptions: 
ASSUMPTION 3.1

(A1) $\widehat{W} \in C^{1}\left([0,1] \times \mathbb{R}_{\text {sym }}^{D \times D} ; \mathbb{R}\right)$.

(A2) $\partial_{\varepsilon} \widehat{W}(\chi, \cdot)$ is strongly monotone uniformly in $\chi$, i.e. there exists a constant $C_{1}>0$ independent of $\chi$ such that for all $\varepsilon_{a}, \varepsilon_{b} \in \mathbb{R}_{\mathrm{sym}}^{D \times D}$,

$$
\left(\partial_{\varepsilon} \widehat{W}\left(\chi, \varepsilon_{a}\right)-\partial_{\varepsilon} \widehat{W}\left(\chi, \varepsilon_{b}\right)\right):\left(\varepsilon_{a}-\varepsilon_{b}\right) \geqslant C_{1}\left|\varepsilon_{a}-\varepsilon_{b}\right|^{2} .
$$

(A3) There exists a constant $C_{2}>0$ such that for all $\chi \in \mathbb{R}, \varepsilon \in \mathbb{R}_{\mathrm{sym}}^{D \times D}$,

$$
\begin{aligned}
|\widehat{W}(\chi, \varepsilon)| & \leqslant C_{2}\left(|\chi|^{2}+|\varepsilon|^{2}+1\right), \\
\left|\partial_{\chi} \widehat{W}(\chi, \varepsilon)\right| & \leqslant C_{2}\left(|\chi|^{2}+|\varepsilon|^{2}+1\right), \\
\left|\partial_{\varepsilon} \widehat{W}(\chi, \varepsilon)\right| & \leqslant C_{2}(|\chi|+|\varepsilon|+1) .
\end{aligned}
$$

These assumptions are satisfied by $\widehat{W}$ under certain conditions:

LeMma 3.2 Let $D<3$ and for $D=2$ let

(1) $\beta^{*}(\chi, \varepsilon)$ be independent of $\varepsilon$, and

(2) $\alpha_{i}$ and $T$ commute whenever $\beta^{*} \in\left\{\gamma^{*}, \beta_{I I}\right\}$.

Then $\widehat{W}$ given by (2.10h) satisfies Assumption 3.1

Proof. (i) In one dimension: From (2.8), (2.10g) and (2.10h) it follows that $\widehat{W} \in C^{1}(\mathbb{R} \times \mathbb{R})$; this verifies (A1). From (2.12),

$$
\partial_{\varepsilon} \varepsilon_{1}^{*}(\chi, \varepsilon)=\frac{\alpha_{2}}{\chi_{2} \alpha_{1}+\chi_{1} \alpha_{2}}, \quad \partial_{\varepsilon} \varepsilon_{2}^{*}(\chi, \varepsilon)=\frac{\alpha_{1}}{\chi_{2} \alpha_{1}+\chi_{1} \alpha_{2}} .
$$

With (2.12a) this yields

$$
\partial_{\varepsilon} \widehat{W}\left(\chi, \varepsilon_{a / b}\right)=\frac{\alpha_{1} \alpha_{2}}{\chi_{2} \alpha_{1}+\chi_{1} \alpha_{2}}\left(\chi_{1}\left(\varepsilon_{1}^{*}\left(\varepsilon_{a / b}\right)-\varepsilon_{1}^{T}\right)+\chi_{2}\left(\varepsilon_{2}^{*}\left(\varepsilon_{a / b}\right)-\varepsilon_{2}^{T}\right)\right)
$$

and therefore

$$
\left(\partial_{\varepsilon} \widehat{W}\left(\chi, \varepsilon_{a}\right)-\partial_{\varepsilon} \widehat{W}\left(\chi, \varepsilon_{b}\right)\right):\left(\varepsilon_{a}-\varepsilon_{b}\right)=\sum_{i=1}^{2} \frac{\chi_{i} \alpha_{i}\left(\varepsilon_{i}^{*}\left(\varepsilon_{a}\right)-\varepsilon_{i}\left(\varepsilon_{b}\right)\right)\left(\varepsilon_{a}-\varepsilon_{b}\right)}{\chi_{2} \alpha_{1}+\chi_{1} \alpha_{2}} .
$$

Assumption (A2) follows since, by (2.12), for $i=1,2$,

$$
\left(\varepsilon_{i}^{*}\left(\varepsilon_{a}\right)-\varepsilon_{i}^{*}\left(\varepsilon_{b}\right)\right)\left(\varepsilon_{a}-\varepsilon_{b}\right) \geqslant c\left|\varepsilon_{a}-\varepsilon_{b}\right|^{2} .
$$

From 2.12], for $i=1,2$ and $\chi \in[0,1]$ we have

$$
\left|\varepsilon_{i}^{*}(\chi, \varepsilon)\right| \leqslant c(|\chi|+|\varepsilon|+1) .
$$

(We denote generic constants by " $c$ ".) Consequently, $\left(\mathrm{A} 3{ }_{1}\right)_{1}$ follows as

$$
\begin{aligned}
|\widehat{W}(\chi, \varepsilon)| & =\left|\chi\left(W_{1}\left(\varepsilon_{1}^{*}\right)-W_{2}\left(\varepsilon_{2}^{*}\right)\right)+W_{2}\left(\varepsilon_{2}^{*}\right)\right| \quad \text { from 2.12a) } \\
& \leqslant c\left(|\chi|\left|W_{1}\left(\varepsilon_{1}^{*}\right)\right|+|\chi|\left|W_{2}\left(\varepsilon_{2}^{*}\right)\right|+1\right) \\
& \leqslant c(|\chi|(|\chi|+|\varepsilon|+1)+1) \quad \text { from 2.12) } \\
& \leqslant c\left(|\chi|^{2}+|\varepsilon|^{2}+1\right) \quad \text { using Young's inequality. }
\end{aligned}
$$


For $\chi \in[0,1]$ we easily compute, for $i=1,2$,

$$
\left|\partial_{\chi} \varepsilon_{i}^{*}(\chi, \varepsilon)\right| \leqslant c(|\chi|+|\varepsilon|+1) .
$$

We use this estimate and Young's inequality in the identity

$$
\partial_{\chi} \widehat{W}(\chi, \varepsilon)=W_{1}\left(\varepsilon_{1}^{*}(\chi, \varepsilon)\right)-W_{2}\left(\varepsilon_{2}^{*}(\chi, \varepsilon)\right)+\sum_{i=1}^{2} \chi_{i} W_{i}^{\prime}\left(\varepsilon_{i}^{*}(\chi, \varepsilon)\right) \partial_{\chi} \varepsilon_{i}^{*}(\chi, \varepsilon)
$$

to obtain

$$
\begin{aligned}
\left|\partial_{\chi} \widehat{W}(\chi, \varepsilon)\right| & \leqslant c\left(\left|\varepsilon_{1}^{*}(\chi, \varepsilon)\right|^{2}+\left|\varepsilon_{2}^{*}(\chi, \varepsilon)\right|^{2}+|\chi|\left(\left|\varepsilon_{1}^{*}(\chi, \varepsilon)\right|+\left|\varepsilon_{2}^{*}(\chi, \varepsilon)\right|\right)+1\right) \\
& \leqslant c\left(|\chi|^{2}+|\varepsilon|^{2}+1\right)
\end{aligned}
$$

which is $\left(\mathrm{A} 3 \mathrm{P}_{2}\right.$.

For $\chi \in[0,1]$,

$$
\begin{aligned}
\left|\partial_{\varepsilon} \widehat{W}(\chi, \varepsilon)\right| & =\left|\frac{\alpha_{1} \alpha_{2}}{\chi_{2} \alpha_{1}+\chi_{1} \alpha_{2}}\right|\left|\chi_{1}\left(\varepsilon_{1}^{*}(\varepsilon)-\varepsilon_{1}^{T}\right)+\chi_{2}\left(\varepsilon_{2}^{*}(\varepsilon)-\varepsilon_{2}^{T}\right)\right| \\
& \leqslant c\left(|\chi|\left|\varepsilon_{1}^{*}\right|+|\chi|\left|\varepsilon_{2}^{*}\right|+1\right) \\
& \leqslant c\left(\left|\varepsilon_{1}^{*}\right|+\left|\varepsilon_{2}^{*}\right|+1\right) \text { as }|\chi| \leqslant 1, \\
& \leqslant c(|\chi|+|\varepsilon|+1) \quad \text { from } 3.1,
\end{aligned}
$$

showing the validity of $(\mathrm{A} 3)_{3}$.

(ii) In two dimensions: The regularity of $\widehat{W}$ is obvious from the definitions. It can be checked directly that $\widehat{W}$ is continuously differentiable as required by $(\mathrm{A} 1$ ).

Since, from [2.10], $\beta^{*} \in\left[0, \gamma^{*}\right]$ and $\gamma^{*}$ is determined solely by $\alpha_{1}, \alpha_{2}$, we have $\left|\beta^{*}\right| \leqslant c$ for a constant $c$ independent of $\chi$ and $\varepsilon$. From that and (2.10g) we obtain

$$
\left|\varepsilon_{i}^{*}\left(\beta^{*}, \chi, \varepsilon\right)\right| \leqslant c(|\chi|+|\varepsilon|+1) .
$$

With $2.8,2.10 \mathrm{~h}$ and $|\chi|^{2} \leqslant 1$ this shows

$$
\begin{aligned}
\left|\beta^{*} \chi_{1} \chi_{2} \operatorname{det}\left(\varepsilon_{2}^{*}(\chi, \varepsilon)-\varepsilon_{1}^{*}(\chi, \varepsilon)\right)\right| & \leqslant c\left(|\chi|^{2}\left(\left|\varepsilon_{1}^{*}(\chi, \varepsilon)\right|^{2}+\left|\varepsilon_{2}^{*}(\chi, \varepsilon)\right|^{2}\right)+1\right) \\
& \leqslant c\left(\left|\varepsilon_{1}^{*}(\chi, \varepsilon)\right|^{2}+\left|\varepsilon_{2}^{*}(\chi, \varepsilon)\right|^{2}\right) \\
& \leqslant c\left(|\chi|^{2}+|\varepsilon|^{2}+1\right)
\end{aligned}
$$

From this we easily verify $\left(A 3{ }_{1}\right.$, as the remaining terms in $2.10 \mathrm{~h}$ can be estimated as in the one-dimensional case.

In order to show $(A 3)_{2}$, we pass through the terms in A.9 (see Appendix A) and notice:

$$
\begin{aligned}
\left|\bar{\sigma}^{*}(\chi, \varepsilon)\right| & =\left|\sum_{i=1}^{2} \chi_{i} \alpha_{i}\left(\varepsilon_{i}^{*}-\varepsilon_{i}^{T}\right)\right| \leqslant c(|\chi|+|\varepsilon|+1) \quad \text { since }|\chi| \leqslant 1, \\
\left|\triangle \varepsilon^{*}(\chi, \varepsilon)\right| & =\left|\varepsilon_{2}^{*}-\varepsilon_{1}^{*}\right| \leqslant c(|\chi|+|\varepsilon|+1),
\end{aligned}
$$




$$
\begin{aligned}
\left|\varphi\left(\triangle \varepsilon^{*}(\chi, \varepsilon)\right)\right| & =\left|\operatorname{det}\left(\triangle \varepsilon^{*}(\chi, \varepsilon)\right)\right| \\
& \leqslant\left|\varepsilon_{2}^{*}(\chi, \varepsilon)-\varepsilon_{1}^{*}(\chi, \varepsilon)\right|^{2} \\
& \leqslant c\left(|\chi|^{2}+|\varepsilon|^{2}+1\right) \quad \text { from 2.10g), } \\
\left|\frac{\partial \beta^{*}}{\partial \chi}(\chi, \varepsilon)\right| & \leqslant c \quad \text { by A.4a } .
\end{aligned}
$$

The terms $W_{i}\left(\varepsilon_{i}^{*}(\chi, \varepsilon)\right), i=1,2$ remaining in A.9 can be estimated as in the one-dimensional case. This verifies $(\mathrm{A} 3)_{2}$.

Now we prove $(\mathrm{A} 2)$. When in Regime III we start from A.12 and find with 3.2 ,

$$
\left|\chi_{1}\left(\alpha_{2}-\gamma^{*} T\right) \alpha^{-1} \alpha_{1}\left(\varepsilon_{1}^{*}-\varepsilon_{1}^{T}\right)\right| \leqslant c\left(|\chi|\left|\varepsilon_{1}^{*}\right|+1\right) \leqslant c\left(\left|\varepsilon_{1}^{*}\right|+1\right) \leqslant c(|\chi|+|\varepsilon|+1) .
$$

In the remaining Regimes 0 , I and II we start from A.11 and the proof is even simpler.

The validation of (A2) relies on equation A.11). By assumption, $\partial \beta^{*} / \partial \varepsilon=0$ and, as $\chi$ is fixed, in $\mathrm{A} .11, \alpha^{-1}$ is a constant tensor. Assumption (A2 follows from

$$
\left(\varepsilon_{1}^{*}\left(\varepsilon_{a}\right)-\varepsilon_{1}^{*}\left(\varepsilon_{b}\right)\right):\left(\varepsilon_{a}-\varepsilon_{b}\right)=\alpha^{-1}\left(\alpha_{2}-\beta^{*} T\right)\left(\varepsilon_{a}-\varepsilon_{b}\right):\left(\varepsilon_{a}-\varepsilon_{b}\right),
$$

a similar equality for $\left(\varepsilon_{2}^{*}\left(\varepsilon_{a}\right)-\varepsilon_{2}^{*}\left(\varepsilon_{b}\right)\right):\left(\varepsilon_{a}-\varepsilon_{b}\right)$, and the positive definiteness of $\left(\alpha_{i}-\beta^{*} T\right)$, $i=1,2$ and $\alpha^{-1}$. The validation of (A2 in Regime III is based directly on A.12).

REMARK 3.3 The critical assumption, (A2), requires the strict convexity of $\widehat{W}(\chi, \cdot)$. But in general $\widehat{W}$ is only quasi-convex except in one dimension where the notions of convexity and quasi-convexity coincide. In higher dimensions the assumption that $\beta^{*}(\chi, \varepsilon)$ is independent of $\varepsilon$ is required to enforce convexity and thus $(\mathrm{A} 2)$. This is satisfied when the solution is globally in (only) one of Regimes 0, I, and III.

\subsection{Existence of weak solutions}

Our main result is the following theorem. The assumption $D<3$ is needed because the proof relies on the explicit computations of $\partial_{\chi} \widehat{W}(\chi, \varepsilon), \partial_{\varepsilon} \widehat{W}(\chi, \varepsilon)$ carried out for $D=1$ in Lemma 3.2 and for $D=2$ in Lemma 3.2 and Sections A.5 and A.6.

Theorem 3.4 Let $D<3$ and $L$ be positive definite. Moreover, for $D=2$, let

(1) $\beta^{*}(\chi, \varepsilon)$ be independent of $\varepsilon$, and

(2) $\alpha_{i}$ and $T$ commute whenever $\beta^{*} \in\left\{\gamma^{*}, \beta_{I I}\right\}$.

Then there exists a weak solution $(\chi, \mu, \mathbf{u})$ to the system 2.1, 2.4, 2.6 such that

(i) $\chi \in C^{0,1 / 4}\left([0, S] ; L^{2}(\Omega)\right)$,

(ii) $\partial_{t} \chi \in L^{2}\left([0, S] ;\left(H^{1}(\Omega)\right)^{\prime}\right)$,

(iii) $\mathbf{u} \in L^{\infty}\left([0, S] ; H^{1}\left(\Omega ; \mathbb{R}^{D}\right)\right)$,

(iv) $\mu \in L^{2}\left([0, S] ; H^{1}(\Omega)\right)$,

(v) $\ln (\chi), \ln (1-\chi) \in L^{1}\left(\Omega_{S}\right)$ and in particular $\chi \in(0,1)$ a.e. in $\Omega$. 
Proof. From Lemma 3.2. under the assumptions of the theorem, $\widehat{W}$ fulfils Assumption 3.1 for all $\chi \in[0,1]$. Let the extension $\widehat{W}_{0}(\cdot, \varepsilon): \mathbb{R} \rightarrow \mathbb{R}$ of $\widehat{W}(\cdot, \varepsilon)$ be given by

$$
\widehat{W}_{0}(\chi, \varepsilon):= \begin{cases}-\chi+1+\widehat{W}(0, \varepsilon) & \text { if } \chi \leqslant-1, \\ \bar{\varphi}(\chi, \varepsilon) & \text { if }-1<\chi<0, \\ \widehat{W}(\chi, \varepsilon) & \text { if } 0 \leqslant \chi \leqslant 1, \\ \bar{\Phi}(\chi, \varepsilon) & \text { if } 1<\chi<2, \\ \chi-1+\widehat{W}(1, \varepsilon) & \text { if } 2 \leqslant \chi .\end{cases}
$$

for suitable functions $\bar{\varphi}, \bar{\Phi}$ that ensure $\widehat{W}_{0}(\cdot, \varepsilon) \in C^{1}(\mathbb{R} ; \mathbb{R})$ together with the validity of (A1) for $\widehat{W}_{0}(\chi, \varepsilon)$ even if $\chi \notin[0,1]$. The construction 3.3 further guarantees that $\widehat{W}_{0}$ is positive and coercive for $|\chi| \rightarrow \infty$.

Now one proves as in [32] the existence of weak solutions to 2.1] for polynomial free energies

$$
\psi(\chi)=\frac{1}{4}(\chi-k)^{2}(\chi-1+k)^{2}
$$

(where $k \in(0,1)$ is a given constant) and $\widehat{W}_{0}$ instead of $\widehat{W}$. The extension $\widehat{W}_{0}$ is necessary as there is no maximum principle for the system, and a polynomial functional $\psi$ (in contrast to 2.4] which is unbounded for $\chi \searrow 0$ and $\chi \nearrow 1$ ) does not ensure $\chi \in[0,1]$.

Then, analogous to [31, Chapter 4] or [33], the existence proof of the first step is reused. With the help of polynomial approximations $\psi_{\delta}$ of (2.4), parametrised by $\delta>0$, where $\psi_{\delta} \rightarrow \psi$, solutions $\left(\chi_{\delta}, \mathbf{u}_{\delta}\right)$ to the regularised system are obtained. Uniform estimates of $\left(\chi_{\delta}, \mathbf{u}_{\delta}\right)$ independent of $\delta$ allow passage to the limit $\delta \searrow 0$. As $\widehat{W}_{0}(\chi, \varepsilon)=\widehat{W}(\chi, \varepsilon)$ for $\chi \in[0,1]$, the proof is complete.

\subsection{Uniqueness of weak solutions}

We begin with some preliminary remarks. Let $\psi=\psi^{1}+\psi^{2}$ for a convex function $\psi^{1}$ and a sublinear function $\partial_{\chi} \psi^{2}$. Let $V:=H^{1}(\Omega), \mathcal{L}: V \rightarrow V^{\prime}$ be given by $\mathcal{L}(\mu)(\zeta):=\int_{\Omega} L \nabla \mu \cdot \nabla \zeta$ and $\mathcal{G}$ be the inverse of $\mathcal{L}$. (Since $L$ is positive definite the existence of $\mathcal{G}$ follows from the Poincaré inequality and the Lax-Milgram theorem.) From this we find that $\mathcal{G}$ is positive definite, self-adjoint, injective and compact. Thus, for all $\zeta \in V$ and $v \in V^{\prime}$,

$$
(L \nabla \mathcal{G} v, \nabla \zeta)_{L^{2}(\Omega)}=\langle\zeta, v\rangle_{V, V^{\prime}}
$$

For $v_{1}, v_{2} \in V^{\prime}$ we define the $L$ scalar product

$$
\left(v_{1}, v_{2}\right)_{L}:=\left(L \nabla \mathcal{G} v_{1}, \nabla \mathcal{G} v_{2}\right)_{L^{2}}
$$

and the corresponding norm $\|v\|_{L}:=\sqrt{(v, v)_{L}}$. Since $v \in V$ canonically defines an element in $V^{\prime}$, $(\cdot, \cdot)_{L}$ and $\|\cdot\|_{L}$ are well defined in $V$. Observe that for $\delta>0$ and $\chi \in V$,

$$
\|\chi\|_{L^{2}}^{2}=(L \nabla \mathcal{G} \chi, \nabla \chi)_{L^{2}} \leqslant\left\|L^{1 / 2} \nabla \mathcal{G} \chi\right\|_{L^{2}}\left\|L^{1 / 2} \nabla \chi\right\|_{L^{2}} \leqslant c \delta^{-1}\|\chi\|_{L}^{2}+\delta\|\nabla \chi\|_{L^{2}}^{2}
$$

(for some constant $c$ ), as a consequence of Young's inequality. We are now ready for:

THEOREM 3.5 Let $D<3$ and $\alpha_{1}=\alpha_{2}$ (i.e., the two phases have identical elastic moduli). Then the weak solution $(\chi, \mu, \mathbf{u})$ of 2.1], 2.4, 2.9) is unique in the spaces stated in Theorem 3.4 
Proof. The following is a modification of the corresponding proof for the case of linear elasticity. Let $\left(\chi^{A}, \mu^{A}, \mathbf{u}^{A}\right),\left(\chi^{B}, \mu^{B}, \mathbf{u}^{B}\right)$ be two weak solutions and let

$$
(\chi, \mu, \mathbf{u})=\left(\chi^{B}, \mu^{B}, \mathbf{u}^{B}\right)-\left(\chi^{A}, \mu^{A}, \mathbf{u}^{A}\right) .
$$

The difference $\chi$ solves

$$
-\int_{\Omega_{S}} \chi \partial_{t} \xi \mathrm{d} x+\int_{\Omega_{S}} L \nabla \mu \cdot \nabla \xi \mathrm{d} x=0
$$

for all $\xi \in L^{2}\left([0, S] ; H^{1}(\Omega)\right)$ with $\xi(S)=0$ and $\partial_{t} \xi \in L^{2}\left(\Omega_{S}\right)$. Pick $t_{0} \in(0, S)$ and $\eta \in$ $L^{2}\left([0, S] ; H^{1}(\Omega)\right)$ and set

$$
\xi(\cdot, t):= \begin{cases}\int_{t}^{t_{0}} \eta(\cdot, s) \mathrm{d} s & \text { if } t \leqslant t_{0}, \\ 0 & \text { if } t>t_{0} .\end{cases}
$$

Because $\partial_{t} \xi=-\eta(t)$ for $t \leqslant t_{0}$ it follows that

$$
0=\int_{\Omega_{t_{0}}} \chi \eta \mathrm{d} x+\int_{\Omega_{t_{0}}} L \nabla \mu \cdot \nabla\left(\int_{t}^{t_{0}} \eta \mathrm{d} s\right) \mathrm{d} x=\int_{\Omega_{t_{0}}} \chi \eta \mathrm{d} x+\int_{\Omega_{t_{0}}} L \nabla\left(\int_{0}^{t} \mu \mathrm{d} s\right) \cdot \nabla \eta \mathrm{d} x .
$$

Therefore we have $\mathcal{G} \chi=-\int_{0}^{t} \mu \mathrm{d} s$ and $\partial_{t} \mathcal{G} \chi=-\mu$. When choosing $\eta:=\mu$ we find, because of $\chi(0)=\mathcal{G} \chi(0)=0$,

$$
-\int_{\Omega_{t_{0}}} \chi \mu \mathrm{d} x=\int_{\Omega_{t_{0}}} L \nabla \mathcal{G} \chi \cdot \nabla \mathcal{G} \chi\left(t_{0}\right) \mathrm{d} x=\frac{1}{2}\left\|\chi\left(\cdot, t_{0}\right)\right\|_{L}^{2} .
$$

For conciseness we set $\varepsilon^{B}:=\varepsilon\left(\mathbf{u}^{B}\right)$ and $\varepsilon^{A}:=\varepsilon\left(\mathbf{u}^{A}\right)$. For the difference $\mu$ we obtain

$$
\begin{aligned}
& \int_{\Omega_{S}} \mu \zeta \mathrm{d} x \\
& \quad=\int_{\Omega_{S}}\left(\gamma \nabla \chi \cdot \nabla \zeta+\left(\psi^{\prime}\left(\chi^{B}\right)-\psi^{\prime}\left(\chi^{A}\right)\right) \zeta+\left(\partial_{\chi} \widehat{W}\left(\chi^{B}, \varepsilon^{B}\right)-\partial_{\chi} \widehat{W}\left(\chi^{A}, \varepsilon^{A}\right)\right) \zeta\right) \mathrm{d} x
\end{aligned}
$$

for all $\zeta \in L^{2}\left([0, S] ; H^{1}(\Omega)\right) \cap L^{\infty}\left(\Omega_{S}\right)$. We choose $\zeta:=\mathcal{X}_{\left[0, t_{0}\right]}\left(\chi^{B}-\chi^{A}\right)$. Since

$$
\left(\partial_{\chi} \psi^{1}\left(\chi^{B}\right)-\partial_{\chi} \psi^{1}\left(\chi^{A}\right)\right)\left(\chi^{B}-\chi^{A}\right) \geqslant 0
$$

it follows that

$$
\begin{aligned}
& \frac{1}{2}\left\|\chi\left(\cdot, t_{0}\right)\right\|_{L}^{2}+\gamma \int_{\Omega_{t_{0}}}|\nabla \chi|^{2} \mathrm{~d} x+\int_{\Omega_{t_{0}}}\left(\partial_{\chi} \widehat{W}\left(\chi^{B}, \varepsilon^{B}\right)-\partial_{\chi} \widehat{W}\left(\chi^{B}, \varepsilon^{A}\right)\right) \chi \mathrm{d} x \\
& \leqslant-\int_{\Omega_{t_{0}}}\left(\partial_{\chi} \psi^{2}\left(\chi^{B}\right)-\partial_{\chi} \psi^{2}\left(\chi^{A}\right)\right) \chi \mathrm{d} x-\int_{\Omega_{t_{0}}}\left(\partial_{\chi} \widehat{W}\left(\chi^{B}, \varepsilon^{A}\right)-\partial_{\chi} \widehat{W}\left(\chi^{A}, \varepsilon^{A}\right)\right) \chi \mathrm{d} x .
\end{aligned}
$$

Because $\alpha_{1}=\alpha_{2}$ we find that $\varphi$ (cf. 2.10e) depends only on $\beta$, thus (cf. Section 2.2)

$$
\beta^{*}\left(\chi^{B}, \varepsilon^{B}\right)=\beta^{*}\left(\chi^{A}, \varepsilon^{A}\right)
$$

and $\alpha$ is a constant matrix. This implies

$$
\varepsilon_{i}^{*}\left(\chi^{B}, \varepsilon^{B}\right)=\varepsilon_{i}^{*}\left(\chi^{B}, \varepsilon^{A}\right), \quad i=1,2 .
$$


By direct inspection, with the above results, we obtain $\partial_{\chi} \widehat{W}\left(\chi^{B}, \varepsilon^{B}\right)-\partial_{\chi} \widehat{W}\left(\chi^{B}, \varepsilon^{A}\right)=0$ and the third integral on the left of 3.8 vanishes identically. The Lipschitz continuity of $\partial_{\chi} \widehat{W}$ and $\psi^{2}$ implies that the integrals on the right hand side of 3.8 can be estimated by $c \int_{\Omega_{t_{0}}}|\chi|^{2} \mathrm{~d} x$ with a constant $c=c\left(\left\|\partial_{\chi} \psi^{2}\right\|_{C^{0,1}},\left\|\partial_{\chi} \widehat{W}\right\|_{C^{0,1}}\right.$ ). With (3.4) and Gronwall's inequality, (3.8) implies $\|\chi\|_{L^{2}}^{2}\left(t_{0}\right)=0$ and thus $\chi=0$.

Taking the difference between the weak formulations of $(2.1 \mathrm{c})$, we find

$$
\int_{\Omega_{S}}\left(\partial_{\varepsilon} \widehat{W}\left(\chi^{B}, \varepsilon^{B}\right)-\partial_{\varepsilon} \widehat{W}\left(\chi^{A}, \varepsilon^{A}\right)\right):\left(\varepsilon^{B}-\varepsilon^{A}\right) \mathrm{d} x=0 .
$$

From A.11 this implies

$$
\int_{\Omega_{t_{0}}}\left(\alpha_{1}-\beta^{*} T\right) \alpha_{1}\left(\varepsilon^{B}-\varepsilon^{A}\right):\left(\varepsilon^{B}-\varepsilon^{A}\right)=0 .
$$

By Korn's inequality, 3.10) yields $\mathbf{u}=0$. With this knowledge, 3.7) finally yields $\mu=0$.

REMARK 3.6 For $\alpha_{1} \neq \alpha_{2}$, the proof of Theorem 3.5 fails as we are unable to control

$$
R:=\int_{\Omega_{t_{0}}}\left(\partial_{\chi} \widehat{W}\left(\chi^{B}, \varepsilon^{B}\right)-\partial_{\chi} \widehat{W}\left(\chi^{B}, \varepsilon^{A}\right)\right)\left(\chi^{B}-\chi^{A}\right) \mathrm{d} x
$$

in 3.8. Even though $\partial_{\chi} \widehat{W}$ is analytic, no powers of $\varepsilon^{B}-\varepsilon^{A}$ can be absorbed on the left of 3.8 when Gronwall's inequality is applied. Also, unlike the linear case, 3.9 ) does not help to show that $R \geqslant 0$. Consequently, the uniqueness of solutions remains open in most cases. In this context we observe that in general the microstructure is not unique.

\section{Numerical simulations}

Many authors favour the solution of the Cahn-Hilliard equation with finite elements: see e.g. [6, 35], the latter containing also a-posteriori error estimators. A finite difference approach is discussed in [27], spectral methods are presented in [20]. Impressive results are presented in [39], where an unconditionally stable non-linear multigrid method that allows for adaptively refined grids is used.

We use finite elements here; a non-linear multigrid method in the spirit of [39] is in preparation; in particular, the multigrid solution of (4.1c) below requires some care.

\subsection{Solution method}

We assume that $\partial \Omega$ is polygonally bounded so as to avoid problems with approximating the boundary. We assume that the triangulation of $\Omega$ is weakly acute, i.e., the sum of the opposite angles relative to any side does not exceed $\pi$ [48].

The $(n+1)^{\text {th }}$ step of the implicit Euler scheme for 2.1 is

$$
\begin{aligned}
\chi^{n+1} & =\chi^{n}+k \operatorname{div}\left(L \nabla \mu^{n+1}\right), \\
\mu^{n+1} & =\psi^{\prime}\left(\chi^{n+1}\right)+\partial_{\chi} \widehat{W}\left(\chi^{n+1}, \varepsilon\left(\mathbf{u}^{n+1}\right)\right)-\gamma \Delta \chi^{n+1}, \\
0 & =\operatorname{div}\left(\partial_{\varepsilon} \widehat{W}\left(\chi^{n+1}, \varepsilon\left(\mathbf{u}^{n+1}\right)\right)\right),
\end{aligned}
$$

with $k>0$ the step size of the time discretisation, $l \in \mathbb{N}, \chi^{l}=\chi(\cdot, l k), \mu^{l}=\mu(\cdot, l k), \mathbf{u}^{l}=\mathbf{u}(\cdot, l k)$. 
This is solved with a conventional predictor-corrector iteration, i.e. for known $\chi^{n}$ a fixed point of the operator implicitly given by (4.1) acting on the triple $\left(\chi^{n+1}, \mu^{n+1}, \mathbf{u}^{n+1}\right)$ is sought.

The splitting of the fourth-order parabolic equation for $\chi$ into the parabolic equation (4.1a) and the elliptic equation $4.1 \mathrm{~b}$, both of second order, permits the use of linear finite elements. Here we focus on $\widehat{W}$ and $4.1 \mathrm{c}$ ) as these aspects are new compared to other models. The computation of $\widehat{W}(\chi, \varepsilon)$ and its derivatives requires, for every vertex of the triangulation,

1. Determination of $\beta^{*}(\chi, \varepsilon)$ and of the regime.

2. Computation of $\varepsilon_{i}^{*}(\chi, \varepsilon)$.

Recall that $\beta^{*} \in\left[0, \gamma^{*}\right]$. As $\gamma^{*}$ depends only on the elastic moduli $\alpha_{1}, \alpha_{2}$, it is computed once at the start of the algorithm; Appendix B presents an explicit formula for $\gamma^{*}$ for cubic materials.

For any spatial point $x \in \Omega$ one needs to compute the regime. This depends on $\varphi, 2.10 \mathrm{f}$. Regime II, where the microstructure is uniquely a rank-one laminate, is numerically the most elaborate case (Section 2.2). Here the unique zero of $\left[0, \gamma^{*}\right] \ni \beta \mapsto \varphi(\beta, \chi, \varepsilon)$ needs to be computed. In our implementation this is done by a Fibonacci subdivision algorithm that splits $\left[0, \gamma^{*}\right]$ consecutively in smaller intervals. Alternatively, this could be realized by a globally convergent Newton method with adapted step size to guarantee that the iterates remain in the feasible range $\left[0, \gamma^{*}\right]$.

In our simulations Regime II, when present, occurred on large subsets of $\Omega$. Thus the computation of $\beta_{I I}$ is time-critical. Significant performance improvement is gained by storing for each lattice point the computed value of $\beta_{I I}$ and using it as the initial guess in the next time step.

Computation of $\varepsilon_{i}^{*}$ and $\beta^{*}$ requires evaluating the inverse of $\chi \alpha_{1}+(1-\chi) \alpha_{2}-\beta T$. Transformation to Mandel notation explained in Appendix $B$ is very helpful here.

Solving (4.1b) and 4.1c) requires explicit expressions for $\partial_{\chi} \widehat{W}(\chi, \varepsilon)$ and $\partial_{\varepsilon} \widehat{W}(\chi, \varepsilon)$, which are presented in (A.9) and (A.11). We remark that the weak formulation of $4.1 \mathrm{c}$ for $\Omega \subset \mathbb{R}^{D}$ is

$$
0=\int_{\Omega} \partial_{\varepsilon} \widehat{W}\left(\chi^{n+1}, \varepsilon\left(\mathbf{u}^{n+1}\right)\right): \varepsilon\left(\mathbf{v}_{j}\right) \mathrm{d} x, \quad 1 \leqslant j \leqslant D N,
$$

with the basis functions for $D=2$,

$$
\left(\mathbf{v}_{i}\right)_{1 \leqslant i \leqslant 2 N}:=\left\{\left(\begin{array}{c}
\varphi_{1} \\
0
\end{array}\right), \ldots,\left(\begin{array}{c}
\varphi_{N} \\
0
\end{array}\right),\left(\begin{array}{c}
0 \\
\varphi_{1}
\end{array}\right), \ldots,\left(\begin{array}{c}
0 \\
\varphi_{N}
\end{array}\right)\right\} .
$$

Here, $\left(\varphi_{i}\right)_{1 \leqslant i \leqslant N}$ denotes the basis functions spanning the finite-dimensional subspace $S^{h} \subset$ $H^{1,2}(\Omega)$ of linear finite elements.

To derive the matrix representation of (4.2) we use $2.10 \mathrm{~g}$ ) and (A.11) to find, for commuting $T$ and $\alpha_{i}$,

$$
\begin{aligned}
\partial_{\varepsilon} \widehat{W}(\chi, \varepsilon)= & G(\beta, \chi) \varepsilon+\chi_{1} \chi_{2} \alpha^{-1}(\beta, \chi)\left(\alpha_{1}-\alpha_{2}\right)\left(I-\lambda \alpha^{-1} T\right)\left(\alpha_{1} \varepsilon_{1}^{T}-\alpha_{2} \varepsilon_{2}^{T}\right) \\
& -\left(\chi_{1} \alpha_{1} \varepsilon_{1}^{T}+\chi_{2} \alpha_{2} \varepsilon_{2}^{T}\right), \\
G(\beta, \chi):= & \chi_{1} \alpha_{1}+\chi_{2} \alpha_{2}-\chi_{1} \chi_{2}\left(\alpha_{1}-\alpha_{2}\right) \alpha^{-1}(\beta, \chi)\left(\alpha_{1}-\alpha_{2}\right) \\
& +\lambda \chi_{1} \chi_{2} \alpha^{-1}(\beta, \chi)\left(\alpha_{1}-\alpha_{2}\right) \alpha^{-1}(\beta, \chi)\left(\alpha_{1}-\alpha_{2}\right) T, \\
\lambda:= & \begin{cases}0 & \text { in Regimes } 0, \mathrm{I}, \mathrm{II}, \\
\gamma^{*} & \text { in Regime III. }\end{cases}
\end{aligned}
$$


The weak formulation (4.2) becomes

$$
\begin{aligned}
& \int_{\Omega} G \varepsilon\left(\mathbf{u}^{n+1}\right): \varepsilon\left(\mathbf{v}_{j}\right) \mathrm{d} x=\int_{\Omega}\left(\chi_{1}^{n+1} \chi_{2}^{n+2} \alpha^{-1}(\beta, \chi)\left(\alpha_{1}-\alpha_{2}\right)\left(\alpha_{1} \varepsilon_{1}^{T}-\alpha_{2} \varepsilon_{2}^{T}\right)\right. \\
& \left.\quad-\chi_{1}^{n+1} \alpha_{1} \varepsilon_{1}^{T}-\chi_{2} \alpha_{2} \varepsilon_{2}^{T}-\lambda \chi_{1}^{n+1} \chi_{2}^{n+1} \alpha^{-1}(\beta, \chi)\left(\alpha_{1}-\alpha_{2}\right) \alpha^{-1} T\left(\alpha_{1} \varepsilon_{1}^{T}-\alpha_{2} \varepsilon_{2}^{T}\right)\right): \varepsilon\left(\mathbf{v}_{j}\right) \mathrm{d} x .
\end{aligned}
$$

We pick $\beta^{*}=\beta^{*}\left(\chi^{n}, \varepsilon^{n}\right)$ when constructing $G$ to avoid a dependence of the matrix on the unknown $\mathbf{u}^{n+1}$. For $n=0$, we choose $\beta^{*} \equiv 0$ as this corresponds to the estimate of the linear model.

Writing $\mathbf{u}^{n+1}=\sum_{i=1}^{2 N} U_{i}^{n+1} \mathbf{v}_{i}$ and $\chi^{n+1}=\sum_{k=1}^{N} d_{k}^{n+1} \varphi_{k}$, we need to compute matrices of the form $\int_{\Omega} G \varepsilon\left(\mathbf{v}_{i}\right): \varepsilon\left(\mathbf{v}_{j}\right) \varphi_{k} \mathrm{~d} x$ and $\int_{\Omega}\left(v: \varepsilon\left(\mathbf{v}_{j}\right)\right) \varphi_{k} \mathrm{~d} x$. Exploiting

$$
\begin{array}{ll}
\varepsilon\left(\mathbf{v}_{j}\right)=\left(\begin{array}{cc}
\partial_{x} \varphi_{j} & \frac{1}{2} \partial_{y} \varphi_{j} \\
\frac{1}{2} \partial_{y} \varphi_{j} & 0
\end{array}\right), & 1 \leqslant j \leqslant N, \\
\varepsilon\left(\mathbf{v}_{j}\right)=\left(\begin{array}{cc}
0 & \frac{1}{2} \partial_{x} \varphi_{j-N} \\
\frac{1}{2} \partial_{x} \varphi_{j-N} & \partial_{y} \varphi_{j-N}
\end{array}\right), & N+1 \leqslant j \leqslant 2 N,
\end{array}
$$

we can apply quadrature formulae to write 4.3 in the matrix form

$$
\mathbf{G}\left(\chi^{n+1}\right) \mathbf{U}^{n+1}=\mathbf{b}\left(\chi^{n+1}\right) \text {. }
$$

Here, $\mathbf{G}\left(\chi^{n+1}\right) \in \mathbb{R}^{2 N \times 2 N}$ and $\mathbf{b} \in \mathbb{R}^{2 N}$ have block structure. A direct calculation shows that in two dimensions

$$
\operatorname{ker} \varepsilon=\left\{\mathbf{v}=\left(v_{1}, v_{2}\right): \mathbb{R}^{2} \rightarrow \mathbb{R}^{2} \mid v_{1}(x, y)=c_{1}-a y, v_{2}(x, y)=c_{2}+a x\right\}
$$

for real constants $c_{1}, c_{2}, a$. Consequently, from (4.3), from $\chi \in(0,1)$ a.e. (Theorem 3.4) and from the positive definiteness of $G_{1}, G_{2}$, it follows that $G$ is singular with $\operatorname{ker} G=\operatorname{ker} \varepsilon$. To solve (4.4) we use a modified conjugate gradient method where the iterates are projected on the orthogonal complement of $\operatorname{ker} G$. This is similar to the case of linearised elasticity; Appendix Chas the details.

\subsection{Sample simulations}

We present three simulations to illustrate features of the new model. We have chosen $\Omega=(0,1)^{2}$, $\theta=0.3, \theta_{c}=1$ and the material constants of one of the materials to be

$$
C_{1,11}=2, \quad C_{1,12}=0, \quad C_{1,44}=1, \quad \varepsilon_{1}^{T}=\left(\begin{array}{ll}
0 & 0 \\
0 & 0
\end{array}\right), \quad w_{1}=0
$$

(see (B.1) for the relationship between $\alpha_{i}$ and $C_{i}$ ).

Simulation 4.1 (Segregation of two cubic materials in Regime II under Neumann BC) We pick the material constants of the second material to be

$$
C_{2,11}=1, \quad C_{2,12}=0, \quad C_{2,44}=5, \quad \varepsilon_{2}^{T}=\left(\begin{array}{cc}
0 & 0 \\
0 & 0.5
\end{array}\right), \quad w_{2}=0 .
$$

Fig. 2 displays the characteristic long-time behaviour of the model. Segregation into two phases (rendered blue and yellow in the pdf file) begins from random initial values around 0.5 for $\chi$; the structure then coarsens with time. The microstructure is computed to be in Regime II always and everywhere.

We observe preferred directions of the phase boundaries going along with a stretching in preferred directions similar to the linear elasticity model. Due to the Neumann boundary conditions, the phase boundaries are always perpendicular to $\partial \Omega$. 


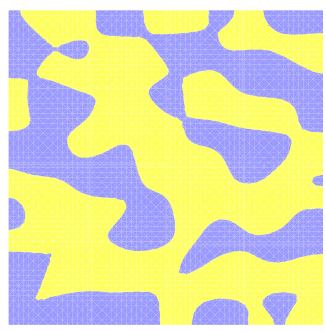

(a) $t=0.07$

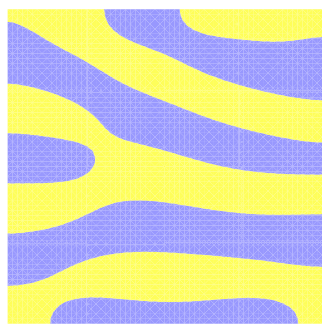

(d) $t=0.72$

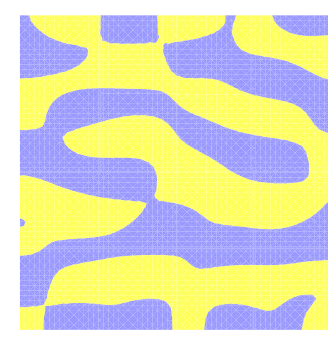

(b) $t=0.16$

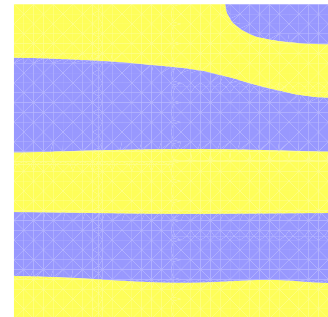

(e) $t=0.94$

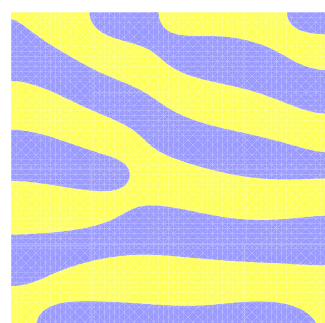

(c) $t=0.29$

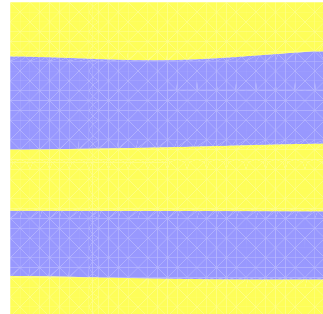

(f) $t=1.5$

FIG. 2. Time evolution of $\chi$ for Simulation 4.1

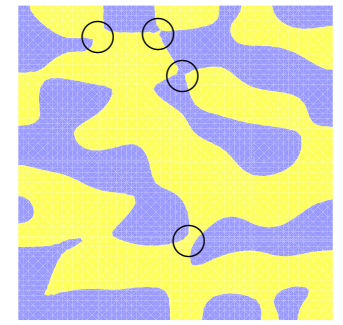

(a) $t=0.10$

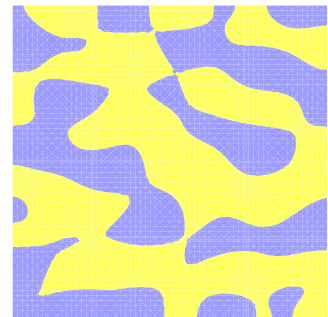

(b) $t=0.101$

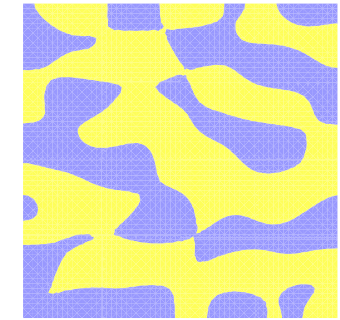

(c) $t=0.102$

FIG. 3. Alternating connectivity at junction points.

Fig. 3 displays further time steps. In the leftmost picture, four circular regions are marked where alternatingly, either the blue phase or the yellow phase is connected. This connectivity changes multiple times during the time evolution. At these junctions, if for a time step e.g. phase 1 is connected, the two unconnected regions of phase 2 are bent towards each other to form a 'checkerboard' geometry. This is observed frequently in our simulations.

Simulation 4.2 (Segregation of two cubic materials under periodic BC) Fig. 4 shows the results of our second calculation, where we re-run Simulation 4.1 with periodic boundary conditions. The coarsening occurs faster but we observe the same global behaviour as before. Besides the altering connectivity at junction points illustrated in Fig. 3, our computations in Fig. 2 and Fig. 4 show another striking difference to the linear model [35]. There it had been found that one phase (the mechanically harder material) forms particles or inclusions that are surrounded by the other simplyconnected phase. 


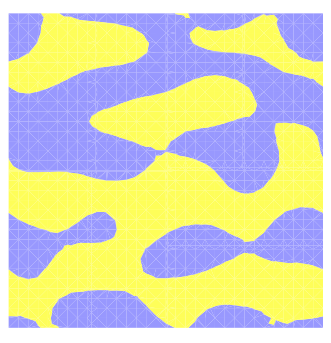

(a) $t=0.18$

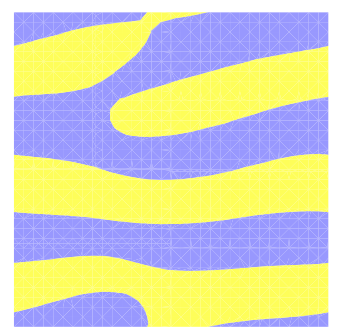

(b) $t=0.77$

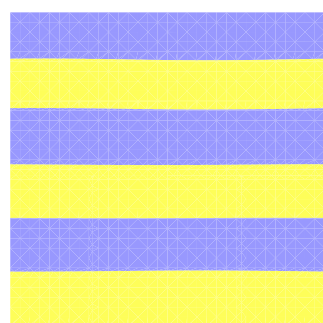

(c) $t=0.90$

FIG. 4. Time evolution of $\chi$ for Simulation 4.2

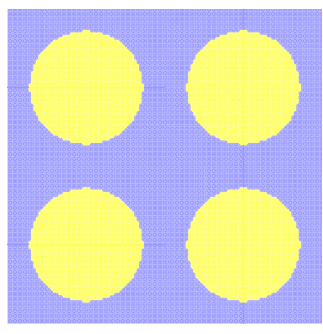

(a) $t=0$

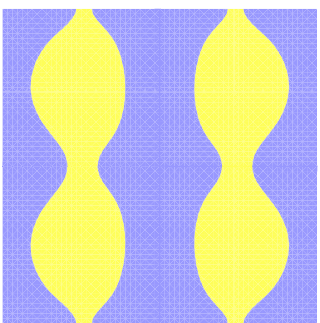

(d) $t=0.03$

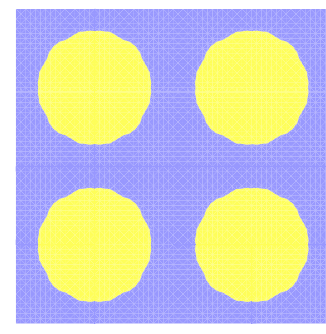

(b) $t=0.002$

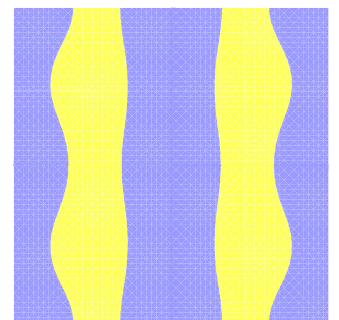

(e) $t=0.07$

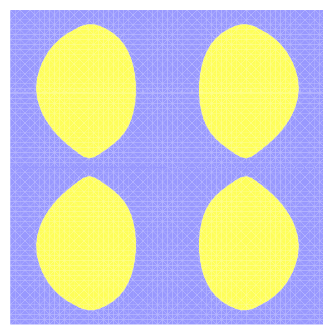

(c) $t=0.02$

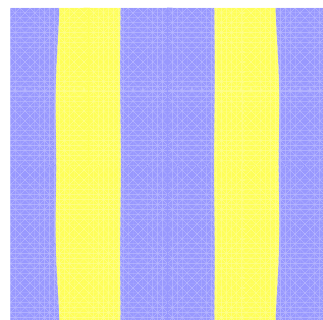

(f) $t=0.2$

FIG. 5. The inclusion problem; see Simulation 4.3

Simulation 4.3 (The inclusion problem) In this simulation we study the inclusion problem further by computing the time evolution in an example where in case of the linear model the initial connectivity changes and inclusion and matrix swap.

The material constants of the second material are

$$
C_{2,11}=1, \quad C_{2,12}=0, \quad C_{2,44}=5, \quad \varepsilon_{2}^{T}=\left(\begin{array}{ll}
1 & 0 \\
0 & 0
\end{array}\right), \quad w_{2}=0 .
$$

Fig. 5 shows the results which confirm our earlier observations. Unlike the behaviour of the linear model [35] the inclusion and matrix phases remain as determined by the initial data. The inclusions are attracted towards each other, unite and stretch. 


\section{Conclusions}

In this article we presented a generalisation of the Cahn-Hilliard model using geometrically linear two-dimensional elasticity which accounts for the microstructure of the solid. We were able to show existence of weak solutions under certain conditions and recover the earlier theory as a special case.

Numerically, the presented solution method is quite inefficient (and thus slow) and was intended only for a preliminary investigation. Nevertheless we discovered the local occurrence, especially in the early stages of the segregation, of alternating connectivities of the phases at junction points. Moreover, unlike the linear model, the harder phase need not form inclusions.

Some key physical limitations of the model are: restriction to geometrically linear elasticity (i.e., linearised kinematics), neglecting surface energy (and thus length scale) in the microstructure, neglect of plastic effects, restriction to two phases, to single crystals and to isothermal situations.

Elasticity is relevant to very many phenomena which have been modelled by numerous well-studied isothermal segregation models and phase-field equations besides the Cahn-Hilliard equation. The approach presented here is a first step in the rigorous incorporation of elasticity in these models.

\section{Appendix A. The derivatives of $\widehat{W}$ in 2D}

For the existence theory of Section 3 we need estimates on $\partial_{\chi} \widehat{W}$ and $\partial_{\varepsilon} \widehat{W}$. These are provided in this appendix. We shall use (without explicit reference) the following result: For $\varepsilon \in \mathbb{R}^{2 \times 2}$ and a linear invertible operator $A: \mathbb{R}^{2 \times 2} \rightarrow \mathbb{R}^{2 \times 2}$,

$$
\begin{aligned}
& (\operatorname{det} \varepsilon)^{\prime}=-(T \varepsilon): \varepsilon^{\prime}, \\
& \left(A^{-1}\right)^{\prime}=-A^{-1} A^{\prime} A^{-1},
\end{aligned}
$$

where ' denotes differentiation and the derivatives are assumed to exist.

\section{A.1 The derivatives of $\varphi$}

From $(2.10 \mathrm{~g})$,

$$
\Delta \varepsilon^{*}(\beta, \chi, \varepsilon)=\alpha^{-1}(\beta, \chi) e(\varepsilon), \quad e(\varepsilon):=\left(\alpha_{1}-\alpha_{2}\right) \varepsilon+\left(\alpha_{2} \varepsilon_{2}^{T}-\alpha_{1} \varepsilon_{1}^{T}\right) .
$$

Then, from 2.10e,

$$
\begin{aligned}
\frac{\partial \varphi}{\partial \chi} & =-\frac{\partial}{\partial \chi} \operatorname{det} \triangle \varepsilon^{*}=T \Delta \varepsilon^{*}: \alpha^{-1} \frac{\partial \alpha}{\partial \chi} \alpha^{-1} e=T \Delta \varepsilon^{*}: \alpha^{-1}\left(\alpha_{2}-\alpha_{1}\right) \triangle \varepsilon^{*} \\
& =T\left(\chi_{2} \alpha_{1}+\chi_{1} \alpha_{2}-\beta T\right)^{-1}\left(\alpha_{2}-\alpha_{1}\right) \triangle \varepsilon^{*}: \Delta \varepsilon^{*} \geqslant 0, \\
\frac{\partial \varphi}{\partial \beta} & =-\frac{\partial}{\partial \beta} \operatorname{det} \triangle \varepsilon^{*}=T \triangle \varepsilon^{*}: \alpha^{-1} \frac{\partial \alpha}{\partial \beta} \alpha^{-1} e=-T \triangle \varepsilon^{*}: \alpha^{-1} T \triangle \varepsilon^{*} \\
& =-\left(\chi_{2} \alpha_{1}+\chi_{1} \alpha_{2}-\beta T\right)^{-1} T \triangle \varepsilon^{*}: T \triangle \varepsilon^{*} \leqslant 0 .
\end{aligned}
$$

In (A3), equality holds only when $\Delta \varepsilon^{*}=0$, in which case $\Delta \varepsilon^{*}(\cdot, \chi, \varepsilon) \equiv 0$, i.e. we are in Regime 0 . Finally, for $f \in \mathbb{R}_{\mathrm{sym}}^{2 \times 2}$,

$$
\frac{\partial \varphi}{\partial \varepsilon}: f=T \alpha^{-1} e: \alpha^{-1} \frac{\partial e}{\partial \varepsilon} f=T \triangle \varepsilon^{*}: \alpha^{-1}\left(\alpha_{1}-\alpha_{2}\right) f=\left(\alpha_{1}-\alpha_{2}\right) \alpha^{-1} T \triangle \varepsilon^{*}: f .
$$


Thus

$$
\frac{\partial \varphi}{\partial \varepsilon}=\left(\alpha_{1}-\alpha_{2}\right) \alpha^{-1} T \triangle \varepsilon^{*}
$$

\section{A.2 The derivatives of $\beta^{*}$}

In Regimes 0 , I and III we immediately obtain $\partial_{\chi} \beta^{*}=0$ and $\partial_{\varepsilon} \beta^{*}=0$. On the other hand, since in Regime II, $\varphi\left(\beta_{I I}(\chi, \varepsilon), \chi, \varepsilon\right)=0$, the chain rule immediately implies

$$
\frac{\partial \beta_{I I}}{\partial \chi}=-\frac{\partial_{\chi} \varphi}{\partial_{\beta} \varphi}, \quad \frac{\partial \beta_{I I}}{\partial \varepsilon}=-\frac{\partial_{\varepsilon} \varphi}{\partial_{\beta} \varphi} .
$$

(From Section A.1. $\partial_{\beta} \varphi \neq 0$ in Regime II.) Using A.3,

$$
\begin{aligned}
& \frac{\partial \beta^{*}}{\partial \chi}= \begin{cases}\frac{\left(T\left(\chi_{2} \alpha_{1}+\chi_{1} \alpha_{2}-\beta T\right)^{-1}\left(\alpha_{2}-\alpha_{1}\right) \Delta \varepsilon^{*}\right): \Delta \varepsilon^{*}}{\left(\left(\chi_{2} \alpha_{1}+\chi_{1} \alpha_{2}-\beta T\right)^{-1}\left(T \Delta \varepsilon^{*}\right)\right): T \Delta \varepsilon^{*}} & \text { in Regime II, } \\
0 & \text { otherwise, }\end{cases} \\
& \frac{\partial \beta^{*}}{\partial \varepsilon}= \begin{cases}\frac{1}{\left(\left(\chi_{2} \alpha_{1}+\chi_{1} \alpha_{2}-\beta T\right)^{-1}\left(T \triangle \varepsilon^{*}\right)\right): T \Delta \varepsilon^{*}}\left(\alpha_{1}-\alpha_{2}\right) \alpha^{-1} T \Delta \varepsilon^{*} & \text { in Regime II, } \\
0 & \text { otherwise. }\end{cases}
\end{aligned}
$$

\section{A.3 The derivatives of $\varepsilon_{i}^{*}$}

From 2.10g,

$\varepsilon_{1}^{*}\left(\beta^{*}(\chi, \varepsilon), \chi, \varepsilon\right)=\alpha^{-1}\left(\beta^{*}(\chi, \varepsilon), \chi\right) e_{1}(\varepsilon), \quad e_{1}(\chi, \varepsilon):=\left(\alpha_{2}-\beta^{*}(\chi, \varepsilon) T\right) \varepsilon-\chi_{2}\left(\alpha_{2} \varepsilon_{2}^{T}-\alpha_{1} \varepsilon_{1}^{T}\right)$, $\varepsilon_{2}^{*}\left(\beta^{*}(\chi, \varepsilon), \chi, \varepsilon\right)=\alpha^{-1}\left(\beta^{*}(\chi, \varepsilon), \chi\right) e_{2}(\varepsilon), \quad e_{2}(\chi, \varepsilon):=\left(\alpha_{1}-\beta^{*}(\chi, \varepsilon) T\right) \varepsilon+\chi_{1}\left(\alpha_{2} \varepsilon_{2}^{T}-\alpha_{1} \varepsilon_{1}^{T}\right)$.

Thus

$$
\begin{aligned}
\frac{\mathrm{d} \varepsilon_{i}^{*}}{\mathrm{~d} \chi} & =-\alpha^{-1} \frac{\mathrm{d} \alpha}{\mathrm{d} \chi} \alpha^{-1} e_{i}+\alpha^{-1} \frac{\mathrm{d} e_{i}}{\mathrm{~d} \chi}=-\alpha^{-1}\left(\frac{\mathrm{d} \alpha}{\mathrm{d} \chi} \varepsilon_{i}^{*}+\frac{\mathrm{d} e_{i}}{\mathrm{~d} \chi}\right), \\
\frac{\mathrm{d} \varepsilon_{i}^{*}}{\mathrm{~d} \varepsilon} & =-\alpha^{-1} \frac{\mathrm{d} \alpha}{\mathrm{d} \varepsilon} \alpha^{-1} e_{i}+\alpha^{-1} \frac{\mathrm{d} e_{i}}{\mathrm{~d} \varepsilon}=-\alpha^{-1}\left(\frac{\mathrm{d} \alpha}{\mathrm{d} \varepsilon} \varepsilon_{i}^{*}+\frac{\mathrm{d} e_{i}}{\mathrm{~d} \varepsilon}\right), \\
\frac{\mathrm{d} \alpha}{\mathrm{d} \chi} & =\frac{\partial \alpha}{\partial \beta^{*}} \frac{\partial \beta^{*}}{\partial \chi}+\frac{\partial \alpha}{\partial \chi}=-T \frac{\partial \beta^{*}}{\partial \chi}+\left(\alpha_{2}-\alpha_{1}\right), \\
\frac{\mathrm{d} e_{i}}{\mathrm{~d} \chi} & =\frac{\partial e_{i}}{\partial \beta^{*}} \frac{\partial \beta^{*}}{\partial \chi}+\frac{\partial e_{i}}{\partial \chi}=-T \varepsilon \frac{\partial \beta^{*}}{\partial \chi}+\left(\alpha_{2} \varepsilon_{2}^{T}-\alpha_{1} \varepsilon_{1}^{T}\right), \\
\frac{\mathrm{d} \alpha}{\mathrm{d} \varepsilon} & =-\frac{\partial \beta^{*}}{\partial \varepsilon} T \\
\frac{\mathrm{d} e_{1}}{\mathrm{~d} \varepsilon} & =-\frac{\partial \beta^{*}}{\partial \varepsilon} T \varepsilon+\alpha_{2}-\beta^{*} T \\
\frac{\mathrm{d} e_{2}}{\mathrm{~d} \varepsilon} & =-\frac{\partial \beta^{*}}{\partial \varepsilon} T \varepsilon+\alpha_{1}-\beta^{*} T .
\end{aligned}
$$


Hence, using 2.10g and $\varepsilon=\chi_{1} \varepsilon_{1}^{*}+\chi_{2} \varepsilon_{2}^{*}$, after some simplification,

$$
\begin{aligned}
\frac{\mathrm{d} \varepsilon_{1}^{*}}{\mathrm{~d} \chi} & =\left(\mathrm{Id}+\chi_{2} \alpha^{-1}\left(\left(\alpha_{2}-\alpha_{1}\right)-\frac{\partial \beta^{*}}{\partial \chi} T\right)\right) \Delta \varepsilon^{*}, \\
\frac{\mathrm{d} \varepsilon_{2}^{*}}{\mathrm{~d} \chi} & =\left(\mathrm{Id}-\chi_{1} \alpha^{-1}\left(\left(\alpha_{2}-\alpha_{1}\right)-\frac{\partial \beta^{*}}{\partial \chi} T\right)\right) \Delta \varepsilon^{*}, \\
\frac{\mathrm{d} \varepsilon_{1}^{*}}{\mathrm{~d} \varepsilon} & =\alpha^{-1}\left(-\chi_{2} \frac{\partial \beta^{*}}{\partial \varepsilon} T \Delta \varepsilon^{*}+\alpha_{2}-\beta^{*} T\right), \\
\frac{\mathrm{d} \varepsilon_{2}^{*}}{\mathrm{~d} \varepsilon} & =\alpha^{-1}\left(\chi_{1} \frac{\partial \beta^{*}}{\partial \varepsilon} T \Delta \varepsilon^{*}+\alpha_{1}-\beta^{*} T\right), \\
\frac{\mathrm{d}}{\mathrm{d} \varepsilon} \Delta \varepsilon^{*} & =\alpha^{-1}\left(\frac{\partial \beta^{*}}{\partial \varepsilon} T \Delta \varepsilon^{*}+\alpha_{1}-\alpha_{2}\right) .
\end{aligned}
$$

A.4 The derivatives of $W_{i}$

From 2.8, $\partial_{\varepsilon} W_{1}=\alpha_{1}\left(\varepsilon-\varepsilon_{1}^{T}\right)$. Using A.5,

$$
\begin{aligned}
\frac{\partial W_{1}\left(\varepsilon_{1}^{*}\right)}{\partial \chi} & =\alpha_{1}\left(\varepsilon_{1}^{*}-\varepsilon_{1}^{T}\right): \frac{\mathrm{d} \varepsilon_{1}^{*}}{\mathrm{~d} \chi} \\
& =\alpha_{1}\left(\varepsilon_{1}^{*}-\varepsilon_{1}^{T}\right):\left(\mathrm{Id}+\chi_{2}\left(\chi_{2} \alpha_{1}+\chi_{1} \alpha_{2}-\beta^{*} T\right)^{-1}\left(\left(\alpha_{2}-\alpha_{1}\right)-\frac{\partial \beta^{*}}{\partial \chi} T\right)\right) \triangle \varepsilon^{*}, \\
\frac{\partial W_{2}\left(\varepsilon_{2}^{*}\right)}{\partial \chi} & =\alpha_{2}\left(\varepsilon_{2}^{*}-\varepsilon_{2}^{T}\right):\left(\mathrm{Id}-\chi_{1}\left(\chi_{2} \alpha_{1}+\chi_{1} \alpha_{2}-\beta^{*} T\right)^{-1}\left(\left(\alpha_{2}-\alpha_{1}\right)-\frac{\partial \beta^{*}}{\partial \chi} T\right)\right) \triangle \varepsilon^{*} .
\end{aligned}
$$

From A.6b,

$$
\begin{aligned}
\frac{\partial}{\partial \varepsilon} W_{1}\left(\varepsilon_{1}^{*}\right) & =\frac{1}{2} \frac{\partial}{\partial \varepsilon}\left(\left(\alpha_{1}\left(\varepsilon_{1}^{*}-\varepsilon_{1}^{T}\right)\right):\left(\varepsilon_{1}^{*}-\varepsilon_{1}^{T}\right)\right) \\
& =\left(\alpha_{1}\left(\varepsilon_{1}^{*}-\varepsilon_{1}^{T}\right)\right): \frac{\partial}{\partial \varepsilon}\left(\varepsilon_{1}^{*}-\varepsilon_{1}^{T}\right) \\
& =-\chi_{2} \frac{\partial \beta^{*}}{\partial \varepsilon}\left(\alpha_{1}\left(\varepsilon_{1}^{*}-\varepsilon_{1}^{T}\right)\right):\left(\alpha^{-1} T \triangle \varepsilon^{*}\right)+\left(\alpha_{1}\left(\varepsilon_{1}^{*}-\varepsilon_{1}^{T}\right)\right):\left(\alpha^{-1}\left(\alpha_{2}-\beta^{*} T\right) \frac{\partial \varepsilon}{\partial \varepsilon}\right) \\
& =-\chi_{2} \frac{\partial \beta^{*}}{\partial \varepsilon}\left(\alpha_{1}\left(\varepsilon_{1}^{*}-\varepsilon_{1}^{T}\right)\right):\left(\alpha^{-1} T \triangle \varepsilon^{*}\right)+\left(\alpha_{2}-\beta^{*} T\right) \alpha^{-1} \alpha_{1}\left(\varepsilon_{1}^{*}-\varepsilon_{1}^{T}\right), \\
\frac{\partial}{\partial \varepsilon} W_{2}\left(\varepsilon_{2}^{*}\right) & =\chi_{1} \frac{\partial \beta^{*}}{\partial \varepsilon}\left(\alpha_{2}\left(\varepsilon_{2}^{*}-\varepsilon_{2}^{T}\right)\right):\left(\alpha^{-1} T \triangle \varepsilon^{*}\right)+\left(\alpha_{1}-\beta^{*} T\right) \alpha^{-1} \alpha_{2}\left(\varepsilon_{2}^{*}-\varepsilon_{2}^{T}\right) .
\end{aligned}
$$

\section{A.5 Computation of $\partial_{\chi} \widehat{W}$}

For $i=1,2$, let

$$
\sigma_{i}^{*}:=\alpha_{i}\left(\varepsilon_{i}^{*}-\varepsilon_{i}^{T}\right), \quad \bar{\sigma}^{*}:=\chi_{1} \sigma_{1}^{*}+\chi_{2} \sigma_{2}^{*}, \quad \Delta \sigma^{*}:=\sigma_{2}^{*}-\sigma_{1}^{*} .
$$


Then, from A.7,

$$
\begin{aligned}
\chi_{1} \frac{\partial W_{1}}{\partial \chi}\left(\varepsilon_{1}^{*}\right) & +\chi_{2} \frac{\partial W_{2}}{\partial \chi}\left(\varepsilon_{2}^{*}\right) \\
& =\bar{\sigma}^{*}: \Delta \varepsilon^{*}-\chi_{1} \chi_{2} \triangle \sigma^{*}:\left(\chi_{2} \alpha_{1}+\chi_{1} \alpha_{2}-\beta T\right)^{-1}\left(\left(\alpha_{2}-\alpha_{1}\right)-\frac{\partial \beta^{*}}{\partial \chi} T\right) \triangle \varepsilon^{*}
\end{aligned}
$$

Also we observe,

$$
\frac{\partial}{\partial \chi}\left(\beta^{*} \chi_{1} \chi_{2} \varphi\left(\Delta \varepsilon^{*}\right)\right)=\chi_{1} \chi_{2} \frac{\partial \beta^{*}}{\partial \chi} \varphi\left(\Delta \varepsilon^{*}\right)+\left(\chi_{2}-\chi_{1}\right) \beta^{*} \varphi\left(\Delta \varepsilon^{*}\right)+\beta^{*} \chi_{1} \chi_{2} \frac{\partial \varphi\left(\Delta \varepsilon^{*}\right)}{\partial \chi} .
$$

We are now ready for the main computation. We find

$$
\begin{aligned}
\frac{\partial \widehat{W}}{\partial \chi}= & \frac{\partial}{\partial \chi}\left(\chi_{1} W_{1}\left(\varepsilon_{1}^{*}\right)+\chi_{2} W_{2}\left(\varepsilon_{2}^{*}\right)-\beta^{*} \chi_{1} \chi_{2} \varphi\left(\Delta \varepsilon^{*}\right)\right) \\
= & \bar{\sigma}^{*}: \Delta \varepsilon^{*}-\chi_{1} \chi_{2} \Delta \sigma^{*}:\left(\chi_{2} \alpha_{1}+\chi_{1} \alpha_{2}-\beta T\right)^{-1}\left(\left(\alpha_{2}-\alpha_{1}\right)-\frac{\partial \beta^{*}}{\partial \chi} T\right) \triangle \varepsilon^{*} \\
& +W_{1}\left(\varepsilon_{1}^{*}\right)-W_{2}\left(\varepsilon_{2}^{*}\right)-\chi_{1} \chi_{2} \frac{\partial \beta^{*}}{\partial \chi} \varphi\left(\Delta \varepsilon^{*}\right)-\left(\chi_{2}-\chi_{1}\right) \beta^{*} \varphi\left(\triangle \varepsilon^{*}\right) \\
& +\beta^{*} \chi_{1} \chi_{2}\left(T\left(\chi_{2} \alpha_{1}+\chi_{1} \alpha_{2}-\beta T\right)^{-1}\left(\alpha_{2}-\alpha_{1}\right) \Delta \varepsilon^{*}\right): \Delta \varepsilon^{*}
\end{aligned}
$$

This identity can be simplified by discussing the different regimes: In Regime 0 , we have $\beta^{*}=$ $\varphi\left(\triangle \varepsilon^{*}\right)=0$ and $\triangle \varepsilon^{*}=\triangle \sigma^{*}=0$. Similarly, in Regime I, we find $\beta^{*}=\partial \beta^{*} / \partial \chi=0$ leading to $\triangle \sigma^{*}=0$. Correspondingly, $\varphi\left(\triangle \varepsilon^{*}\right)=0$ in Regime II. Finally $\beta^{*}=\gamma^{*}$ and $\partial \beta^{*} / \partial \chi=0$ in Regime III. Putting everything together, this yields

$$
\frac{\mathrm{d} \widehat{W}}{\mathrm{~d} \chi}(\chi, \varepsilon)=\bar{\sigma}^{*}: \Delta \varepsilon^{*}+W_{1}\left(\varepsilon_{1}^{*}\right)-W_{2}\left(\varepsilon_{2}^{*}\right)+ \begin{cases}0 & \text { in Regimes } 0 \text { and I, } \\ \beta^{*} \chi_{1} \chi \chi_{2} \frac{\partial \beta^{*}}{\partial \chi}\left\|\Delta \varepsilon^{*}\right\|^{2} & \text { in Regime II, } \\ \left(\chi_{1}-\chi_{2}\right) \gamma^{*} \varphi\left(\Delta \varepsilon^{*}\right) & \text { in Regime III. }\end{cases}
$$

\section{A.6 Computation of $\partial_{\varepsilon} \widehat{W}$}

Using A.8,

$$
\begin{aligned}
\frac{\partial \widehat{W}}{\partial \varepsilon}(\chi, \varepsilon)= & \frac{\partial}{\partial \varepsilon}\left(\chi_{1} W_{1}\left(\varepsilon_{1}^{*}\right)+\chi_{2} W_{2}\left(\varepsilon_{2}^{*}\right)+\beta^{*} \chi_{1} \chi_{2} \operatorname{det} \Delta \varepsilon^{*}\right) \\
= & \chi_{1} \chi_{2}\left(\left(\alpha_{2}\left(\varepsilon_{2}^{*}-\varepsilon_{2}^{T}\right)-\alpha_{1}\left(\varepsilon_{1}^{*}-\varepsilon_{1}^{T}\right)\right):\left(\alpha^{-1} T \Delta \varepsilon^{*}\right)+\operatorname{det} \Delta \varepsilon^{*}\right) \frac{\partial \beta^{*}}{\partial \varepsilon} \\
& +\chi_{1}\left(\alpha_{2}-\beta^{*} T\right) \alpha^{-1} \alpha_{1}\left(\varepsilon_{1}^{*}-\varepsilon_{1}^{T}\right)+\chi_{2}\left(\alpha_{1}-\beta^{*} T\right) \alpha^{-1} \alpha_{2}\left(\varepsilon_{2}^{*}-\varepsilon_{2}^{T}\right)
\end{aligned}
$$

This can be simplified further when $\alpha_{i}$ and $T$ commute (which is the case, for example, when $\alpha_{1}$ and $\alpha_{2}$ correspond to cubic materials). As the calculations are involved, we formulate the result as a lemma: 
LEMMA A. 1 Let $\alpha_{i}$ and $T$ commute. Then

$$
\begin{aligned}
\frac{\partial \widehat{W}}{\partial \varepsilon}(\chi, \varepsilon)= & \chi_{1} \alpha_{1}\left(\varepsilon_{1}^{*}-\varepsilon_{1}^{T}\right)+\chi_{2} \alpha_{2}\left(\varepsilon_{2}^{*}-\varepsilon_{2}^{T}\right) \\
& + \begin{cases}\gamma^{*} \chi_{1} \chi_{2} \alpha^{-1}\left(\gamma^{*}, \chi\right)\left(\alpha_{1}-\alpha_{2}\right) T\left(\varepsilon_{2}^{*}-\varepsilon_{1}^{*}\right) & \text { in Regime III, } \\
0 & \text { else. }\end{cases}
\end{aligned}
$$

Alternatively, in Regime III,

$$
\begin{aligned}
\frac{\partial \widehat{W}}{\partial \varepsilon}(\chi, \varepsilon)= & \chi_{1}\left(\alpha_{2}-\gamma^{*} T\right) \alpha^{-1}\left(\gamma^{*}, \chi\right) \alpha_{1}\left(\varepsilon_{1}^{*}\left(\gamma^{*}, \chi, \varepsilon\right)-\varepsilon_{1}^{T}\right) \\
& +\chi_{2}\left(\alpha_{1}-\gamma^{*} T\right) \alpha^{-1}\left(\gamma^{*}, \chi\right) \alpha_{2}\left(\varepsilon_{2}^{*}\left(\gamma^{*}, \chi, \varepsilon\right)-\varepsilon_{2}^{T}\right) .
\end{aligned}
$$

Proof. In Regimes 0, I and III, $\partial \beta^{*} / \partial \varepsilon=0$, and we immediately obtain A.12 from A.10. Moreover, in Regime II, $\beta^{*}=\beta_{I I}$ is the unique root of $\varphi$ and, from (2.10e),

$$
\varphi\left(\beta^{*}, \chi, \varepsilon\right)=-\operatorname{det}\left(\Delta \varepsilon^{*}\left(\beta^{*}, \chi, \varepsilon\right)\right)=0 .
$$

Thus, in all four regimes,

$$
\left(\operatorname{det} \Delta \varepsilon^{*}\right) \frac{\partial \beta^{*}}{\partial \varepsilon}=0
$$

Based on the equations

$$
\begin{aligned}
\Delta \varepsilon^{*}(\varepsilon) & =\alpha^{-1}\left(\left(\alpha_{1}-\alpha_{2}\right) \varepsilon-\left(\alpha_{1} \varepsilon_{1}^{T}-\alpha_{2} \varepsilon_{2}^{T}\right)\right), \\
\varepsilon_{2}^{*}(\varepsilon)-\varepsilon_{2}^{T} & =\alpha^{-1}\left(\left(\alpha_{1}-\beta^{*} T\right)\left(\varepsilon-\varepsilon_{2}^{T}\right)+\chi_{1} \alpha_{1}\left(\varepsilon_{2}^{T}-\varepsilon_{1}^{T}\right)\right), \\
\varepsilon_{1}^{*}(\varepsilon)-\varepsilon_{1}^{T} & =\alpha^{-1}\left(\left(\alpha_{2}-\beta^{*} T\right)\left(\varepsilon-\varepsilon_{1}^{T}\right)-\chi_{2} \alpha_{2}\left(\varepsilon_{2}^{T}-\varepsilon_{1}^{T}\right)\right), \\
\alpha_{2}\left(\varepsilon_{2}^{*}(\varepsilon)-\varepsilon_{2}^{T}\right)-\alpha_{1}\left(\varepsilon_{1}^{*}(\varepsilon)-\varepsilon_{1}^{T}\right) & =\beta^{*} \alpha^{-1} T\left(\left(\alpha_{1}-\alpha_{2}\right) \varepsilon+\left(\alpha_{2} \varepsilon_{2}^{T}-\alpha_{1} \varepsilon_{1}^{T}\right)\right)
\end{aligned}
$$

we obtain for the first term in A.10, while in Regime II,

$$
\begin{aligned}
\chi_{1} \chi_{2}\left(\left(\alpha_{2}\left(\varepsilon_{2}^{*}-\varepsilon_{2}^{T}\right)-\right.\right. & \left.\left.\alpha_{1}\left(\varepsilon_{1}^{*}-\varepsilon_{1}^{T}\right)\right):\left(\alpha^{-1} T \triangle \varepsilon^{*}\right)\right) \frac{\partial \beta^{*}}{\partial \varepsilon} \\
& =-\chi_{1} \chi_{2}\left(\frac{\beta^{*} T\left(B \varepsilon-\alpha^{-1} s\right): \alpha^{-1} T\left(B \varepsilon-\alpha^{-1} s\right)}{\alpha^{-1} T\left(B \varepsilon-\alpha^{-1} s\right): T\left(B \varepsilon-\alpha^{-1} s\right)}\right) T B\left(B \varepsilon-\alpha^{-1} s\right) \\
& =-\beta^{*} \chi_{1} \chi_{2} T B\left(B \varepsilon-\alpha^{-1} s\right)
\end{aligned}
$$

where, for conciseness, we have set

$$
B(\beta, \chi):=\alpha^{-1}(\beta, \chi)\left(\alpha_{1}-\alpha_{2}\right), \quad s:=\alpha_{1} \varepsilon_{1}^{T}-\alpha_{2} \varepsilon_{2}^{T} .
$$

Then A.10 becomes

$$
\begin{aligned}
\frac{\partial \widehat{W}}{\partial \varepsilon}(\chi, \varepsilon)= & \chi_{1}\left(\alpha_{2}-\beta^{*} T\right) \alpha^{-1} \alpha_{1}\left(\varepsilon_{1}^{*}-\varepsilon_{1}^{T}\right)+\chi_{2}\left(\alpha_{1}-\beta^{*} T\right) \alpha^{-1} \alpha_{2}\left(\varepsilon_{2}^{*}-\varepsilon_{2}^{T}\right) \\
& + \begin{cases}-\beta^{*} \chi_{1} \chi_{2} T B\left(\varepsilon_{2}^{*}-\varepsilon_{1}^{*}\right) & \text { in Regime II } \\
0 & \text { else. }\end{cases}
\end{aligned}
$$


Now,

$$
\alpha_{2}-\beta^{*} T=\alpha-\chi_{2}\left(\alpha_{1}-\alpha_{2}\right), \quad \alpha_{1}-\beta^{*} T=\alpha+\chi_{1}\left(\alpha_{1}-\alpha_{2}\right) .
$$

Inserting these identities in A.15, we obtain

$$
\begin{aligned}
\frac{\partial \widehat{W}}{\partial \varepsilon}(\chi, \varepsilon)= & \chi_{1} \alpha_{1}\left(\varepsilon_{1}^{*}-\varepsilon_{1}^{T}\right)+\chi_{2} \alpha_{2}\left(\varepsilon_{2}^{*}-\varepsilon_{2}^{T}\right)+\chi_{1} \chi_{2}\left(\alpha_{1}-\alpha_{2}\right) \alpha^{-1}\left[\alpha_{2}\left(\varepsilon_{2}^{*}-\varepsilon_{2}^{T}\right)-\alpha_{1}\left(\varepsilon_{1}^{*}-\varepsilon_{1}^{T}\right)\right] \\
& + \begin{cases}-\beta^{*} \chi_{1} \chi_{2} T \alpha^{-1}(\beta, \chi)\left(\alpha_{1}-\alpha_{2}\right)\left(\varepsilon_{2}^{*}-\varepsilon_{1}^{*}\right) & \text { in Regime II, } \\
0 & \text { else. }\end{cases}
\end{aligned}
$$

In Regime 0 or I, $\beta^{*}=0$ and $(\mathrm{A} .16]$ leads to $(\mathrm{A} .11]$, as by $(\mathrm{A} .14]$ the term within [...] also disappears. When in Regime II, based on A.13, A.14 we can rewrite the last term A.16) and for commuting $T, B$ and $\alpha_{1}-\alpha_{2}$, the first and the last term in (A.16) cancel out. Finally, in Regime III, the first term in $\mathrm{A} .16$ is not there and rearranging yields $\mathrm{A} .11$.

\section{Appendix B. Computation of $\gamma^{*}$ for cubic materials}

Let $\tilde{\sim}: \mathbb{R}_{\text {sym }}^{2 \times 2} \rightarrow \mathbb{R}^{3}$ be the isometry given by

$$
\varepsilon:=\left(\begin{array}{ll}
\varepsilon_{11} & \varepsilon_{12} \\
\varepsilon_{12} & \varepsilon_{22}
\end{array}\right) \mapsto \tilde{\varepsilon}:=\left(\varepsilon_{11}, \varepsilon_{22}, \sqrt{2} \varepsilon_{12}\right) .
$$

(This scaled variant of the Voigt notation goes back to Mandel [50].) The orthonormal basis of $\mathbb{R}_{\mathrm{sym}}^{2 \times 2}$,

$$
\left\{\left(\begin{array}{ll}
1 & 0 \\
0 & 0
\end{array}\right),\left(\begin{array}{ll}
0 & 0 \\
0 & 1
\end{array}\right),\left(\begin{array}{cc}
0 & 1 / \sqrt{2} \\
1 / \sqrt{2} & 0
\end{array}\right)\right\}
$$

is mapped to the standard basis of $\mathbb{R}^{3}$ and cubic elastic moduli to

$$
\begin{aligned}
\left(\begin{array}{ccc}
C_{11} & C_{12} & 0 \\
C_{12} & C_{11} & 0 \\
0 & 0 & 2 C_{44}
\end{array}\right) & =U\left(\begin{array}{ccc}
C_{11}-C_{12} & 0 & 0 \\
0 & C_{11}+C_{12} & 0 \\
0 & 0 & 2 C_{44}
\end{array}\right) U^{t}, \\
U & :=\left(\begin{array}{ccc}
1 / \sqrt{2} & -1 / \sqrt{2} & 0 \\
1 / \sqrt{2} & 1 / \sqrt{2} & 0 \\
0 & 0 & 1
\end{array}\right) .
\end{aligned}
$$

Here $C_{11}, C_{12}$ and $C_{44}$ are the usual cubic elastic constants. Moreover, $T$ has the representation

$$
\left(\begin{array}{ccc}
0 & -1 & 0 \\
-1 & 0 & 0 \\
0 & 0 & 1
\end{array}\right)=U\left(\begin{array}{ccc}
1 & 0 & 0 \\
0 & -1 & 0 \\
0 & 0 & 1
\end{array}\right) U^{t} .
$$

With this it is easy to see that the quantity $\gamma$ in 2.10b is given by

$$
\gamma:=\min \left\{C_{11}-C_{12}, 2 C_{44}\right\}
$$




\section{Appendix C. Projection on the orthogonal complement of ker $\varepsilon$}

To implement the conjugate gradient method and to solve (4.4) we apply an orthogonal projection $P$ that maps the iterates to $(\operatorname{ker} \varepsilon)^{\perp}$ (see below). The modified conjugate gradient method for the system $G U^{n+1}=b$ reads as follows:

- Start: Choose $U_{0}^{n+1}$.

- Project initial value: Replace $U_{0}^{n+1}$ by $P U_{0}^{n+1}$.

- Set $r_{0}=P\left(G U_{0}^{n+1}-b\right)$ and $d_{1}=-r_{0}$.

- Iterate for $j=1,2,3, \ldots$

$$
\begin{gathered}
\text { if } j>1\left\{\begin{array}{l}
\alpha_{j-1}=\frac{\left(r_{j-1}, G d_{j-1}\right)}{\left(d_{j-1}, G d_{j-1}\right)}, \\
d_{j}=-r_{j-1}+\alpha_{j-1} d_{j-1},
\end{array}\right. \\
q_{j}=P\left(G d_{j}\right), \quad \beta_{j}=\frac{\left(r_{j-1}, d_{j}\right)}{\left(d_{j}, q_{j}\right)}, \quad U_{j}^{n+1}=U_{j-1}^{n+1}+\beta_{j} d_{j}, \quad r_{j}=r_{j-1}+\beta_{j} q_{j} .
\end{gathered}
$$

In practise the algorithm even works without projections as numerically the $U_{j}^{n+1}$ are never exactly orthogonal to $\operatorname{ker} G$. Yet, replacing $U_{0}^{n+1}$ by $P U_{0}^{n+1}$ is advantageous as for an initial value with a small component in $\operatorname{ker} G$, the standard conjugate gradient method needs many iterations.

To define $P$ we equip

$$
\mathcal{W}:=\left\{w: \Omega \rightarrow \mathbb{R}^{2} \mid w \text { is affine }\right\}
$$

with the Euclidean inner product

$$
\left\langle\left(a_{1}, a_{2}\right),\left(b_{1}, b_{2}\right)\right\rangle:=\int_{\Omega}\left(a_{1}(x, y) b_{1}(x, y)+a_{2}(x, y) b_{2}(x, y)\right) \mathrm{d} x \mathrm{~d} y .
$$

It is elementary to check that

$$
\begin{array}{ll}
w_{1}(x, y):=(1,0), & w_{2}(x, y):=(0,1), \\
w_{3}(x, y):=\frac{1}{\sqrt{6}}\left(-y+\frac{1}{2}, x-\frac{1}{2}\right), & w_{4}(x, y):=\frac{1}{\sqrt{12}}\left(x-\frac{1}{2}, 0\right), \\
w_{5}(x, y):=\frac{1}{\sqrt{6}}\left(y-\frac{1}{2}, x-\frac{1}{2}\right), & w_{6}(x, y):=\frac{1}{\sqrt{12}}\left(0, y-\frac{1}{2}\right)
\end{array}
$$

form an orthonormal basis of $(\mathcal{W},\langle\cdot, \cdot\rangle)$. The orthogonal complement of ker $\varepsilon$ is taken with respect to $\langle\cdot, \cdot\rangle$. Since $\varepsilon_{11}((-y, x))=\varepsilon_{22}((-y, x))=\varepsilon_{12}((-y, x))=0$, we find

$$
\operatorname{ker} \varepsilon=\operatorname{span}\left\{w_{1}, w_{2}, w_{3}\right\}, \quad(\operatorname{ker} \varepsilon)^{\perp}=\operatorname{span}\left\{w_{4}, w_{5}, w_{6}\right\} .
$$

Note that

$$
\left\{v_{1}=w_{1}, v_{2}=w_{2}, v_{3}(x, y)=(x, 0), v_{4}(x, y)=(0, x), v_{5}(x, y)=(y, 0), v_{6}(x, y)=(0, y)\right\}
$$

also form a basis of $\mathcal{W}$. Expressing $v$ s in terms of $w \mathrm{~s}$, we obtain the following formula for $P$ :

$$
\begin{aligned}
P \sum_{i=1}^{6} \beta_{i} v_{i} & =\sqrt{12} \beta_{3} w_{4}+\frac{\sqrt{6}}{2}\left(\beta_{4}+\beta_{5}\right) w_{5}+\sqrt{12} \beta_{6} w_{6} \\
& =-\left(\frac{\beta_{3}}{2}+\frac{\beta_{4}}{4}+\frac{\beta_{5}}{4}\right) v_{1}-\left(\frac{\beta_{4}}{4}+\frac{\beta_{5}}{4}+\frac{\beta_{6}}{2}\right) v_{2}+\beta_{3} v_{3}+\frac{\beta_{4}+\beta_{5}}{2}\left(v_{4}+v_{5}\right)+\beta_{6} v_{6} .
\end{aligned}
$$




\section{REFERENCES}

1. Akaiwa, N., Thornton, K., \& Voorhees, P. W. Dynamics of late-stage phase separation in crystalline solids. Phys. Rev. Lett. 86 (2001), 1259-1263.

2. Alikakos, N. D., Bates, P. W., \& Chen, X. Convergence of the Cahn-Hilliard equation to the HeleShaw model. Arch. Ration. Mech. Anal. 128 (1994), 165-205. Zbl 0828.35105 MR 1308851

3. Alt, H. W., \& PAWŁOW, I. A mathematical model of dynamics of nonisothermal phase separation. Phys. D 59 (1992), 389-416. Zbl 0763.58031 MR 1192751

4. Alt, H. W., \& PaWŁow, I. On the entropy principle of phase transition models with a conserved order parameter. Adv. Math. Sci. Appl. 6 (1996), 291-376. Zbl 0855.35122 MR 1385773

5. Bänsch, E., Morin, P., \& Nochetto, R. H. A finite element method for surface diffusion: The parametric case. J. Comput. Phys. 203 (2005), 321-343. Zbl 1070.65093 MR 2104399

6. Barrett, J., \& Blowey, J. F. Finite element approximation of an Allen-Cahn/Cahn-Hilliard system. IMA J. Numer. Anal. 22 (2002), 11-71. Zbl 1036.76030 MR 1880052

7. Barrett, J. W., Garcke, H., \& NÜrnberg, R. A parametric finite element method for fourth order geometric evolution equations. J. Comput. Phys. 222 (2007), 441-467. Zbl 1112.65093 MR 2298053

8. Bhattacharya, K. Comparison of the geometrically nonlinear and linear theories of martensitic transformation. Contin. Mech. Thermodynam. 5 (1993), 205-242. Zbl 0780.73005 MR 1236099

9. Bhattacharya, K. Microstructure of Martensite. Oxford Univ. Press (2003). Zbl 1109.74002 MR 2282631

10. Blesgen, T. A generalization of the Navier-Stokes equations to two-phase flows. Phys. D 32 (1999), 1119-1123.

11. Blesgen, T., Luckhaus, S., \& Bente, K. Diffusion induced segregation in the ternary case of sphalerite, chalcopyrite and cubanite. Crystal Res. Tech. 39 (2004), 969-979.

12. Blesgen, T., \& SChlömerkemper, A. A counter example to weak maximum principles for locally vanishing elliptic operators. Preprint Max-Planck Institute Math. Sciences 18, Leipzig (2010).

13. Cahn, J. W., Elliott, C. M., \& Novick-Cohen, A. The Cahn-Hilliard equation with a concentration dependent mobility: Motion by minus the Laplacian of the mean curvature. Eur. J. Appl. Math. 7 (1996), 287-301. Zbl 0861.35039 MR 1401172

14. Cahn, J. W., \& Hilliard, J. E. Free energy of a non-uniform system I. Interfacial free energy. $J$. Chem. Phys. 28 (1958), 258-267.

15. CAhn, J. W., \& LARChÉ, F. C. The effect of self-stress on diffusion in solids. Acta Metall. 30 (1982), $1835-1845$.

16. Cahn, J. W., \& Novick-Cohen, A. Limiting motion for an Allen-Cahn/Cahn-Hilliard system. In: Conference on Free Boundary Problems, Theory and Applications (Zakopane, 1995), Longman (1996), 89-97. Zbl 0865.35145 MR 1462972

17. Chen, X. Global asymptotic limit of the Cahn-Hilliard equation. J. Differential Geom. 44 (1996), 262311. Zbl 0874.35045 MR 1425577

18. Chenchiah, I. V., \& Bhattacharya, K. The relaxation of two-well energies with possibly unequal moduli. Arch. Ration. Mech. Anal. 187 (2008), 409-479. Zbl $1134.74042 \mid$ MR 2372811

19. Clarenz, U., DZIUK, G., \& RumpF, M. On generalized mean curvature flow in surface processing. In: Geometric Analysis and Nonlinear Partial Differential Equations, Springer, Berlin (2003), 217-248. Zbl 1035.53091 MR 2008341

20. Copetti, M. I. M. Numerical analysis of spectral approximations to the Cahn-Hilliard equation. Comput. Appl. Math. 19 (2000), 307-321. Zbl 1156.65316 MR 1995201

21. Dacorogna, B. Direct Methods in the Calculus of Variations. 2nd ed., Springer, Berlin (2008). Zbl 1140.49001 MR 2361288 
22. Dal Paso, R., Giacomelli, L., \& GRÜN, G. A waiting time phenomenon for thin films. Ann. Scuola Norm. Sup. Pisa 30 (2001), 437-463. Zbl 1024.35051 MR 1895718

23. Dreyer, W., \& Müller, H. W. A study of the coarsening in tin/lead solders. J. Solids Structures 37 (2000), 3841-3871. Zbl 0973.74059

24. Dreyer, W., \& MüLler, H. W. Modeling diffusional coarsening in eutectic tin: lead solders. J. Solids Structures 38 (2001), 1433-1458. Zbl 0961.74523

25. Elliott, C. M., \& Luckhaus, S. A generalized diffusion equation for phase separation of a multicomponent mixture with interfacial free energy. University of Minnesota Preprint 887 (1991).

26. Eshelby, J. D. Elastic inclusions and inhomogeneities, Progr. Solid Mech. 2 (1961), 89-140. MR 0134510

27. EYRE, D. J. Unconditionally gradient stable time marching the Cahn-Hilliard equation. In: Computational and Mathematical Models of Microstructural Evolution (San Francisco, 1998), Mater. Res. Soc., Warrendale, PA (1998), 39-46. MR 1676409

28. Fong, C. Y., Weber, W., \& Phillips, J. C. Violation of Vegard's law in covalent semiconductor alloys. Phys. Rev. B 14 (1976), 5387-5391

29. FRIED, E., \& GRACH, G. An order-parameter based theory as a regularization of a sharp-interface theory for solid-solid phase transitions. Arch. Ration. Mech. Anal. 138 (1997), 355-404. Zbl 0910.73006 MR 1467559

30. Fried, E., \& Gurtin, M. E. Coherent solid-state phase transitions with atomic diffusion: A thermodynamical treatment. J. Statist. Phys. 95 (2004), 1361-1427. Zbl 0952.74053 MR 1712451

31. GARCKE, H. On mathematical models for phase separation in elastically stressed solids. habilitation thesis, Univ. of Bonn (2000).

32. Garcke, H. On Cahn-Hilliard systems with elasticity. Proc. Roy. Soc. Edinburgh Sect. A 133 (2003), 307-331. Zbl 1130.74037 MR 1969816

33. GARCKe, H. On a Cahn-Hilliard model for phase separation with elastic misfit. Ann. Inst. H. Poincaré Anal. Non Linéaire 22 (2005), 165-185. Zbl 1072.35081 MR 2124161

34. Garcke, H., Nestler, B., \& Stinner, B. A diffuse interface model for alloys with multiple components and phases. SIAM J. Appl. Math. 64 (2004), 775-799. Zbl 1126.82025 MR 2068122

35. Garcke, H., RumpF, M., \& Weikard, U. The Cahn-Hilliard equation with elasticity-finite element approximation and qualitative studies. Interfaces Free Bound. 3 (2001), 101-118. Zbl 0972.35164 MR 1805080

36. Gurtin, M. E. Generalized Ginzburg-Landau and Cahn-Hilliard equations based on a microforce balance. Phys. D 92 (1996), 178-192. Z Zbl 0885.35121 MR 1387065

37. Jou, H. J., Leo, P. H., \& Lowengrub, J. S. Microstructural evolution in inhomogeneous elastic media. J. Comput. Phys. 131 (1997), 109-148. Zbl 0880.73050

38. KAWASAKI, K., \& ENOMOto, Y. Statistical theory of Ostwald ripening with elastic field interactions. Phys. A 150 (1988), 463-498.

39. KAY, D., \& Welford, R. A multigrid finite element solver for the Cahn-Hilliard equation. J. Comput. Phys. 212 (2006), 288-304. Zbl 1081.65091 MR 2183612

40. Khachaturyan, A. G., Semenovskaya, S. V., \& Morris, J. W. Theoretical analysis of straininduced shape changes in cubic precipitates during coarsening. Acta Metall. 36 (1988), 1563-1572.

41. Kohn, R. V., \& Vogelius, M. Relaxation of a variational method for impedance computed tomography. Comm. Pure Appl. Math. 60 (1987), 745-777. Zbl 0659.49009 MR 0910952

42. LARChÉ, F. C., \& CAHn, J. C. The interaction of composition and stress in crystalline solids. Acta Metall. 33 (1985), 331-357.

43. Leo, P., Lowengrub, J. S., \& Nie, Q. Microstructural evolution in orthorhombic elastic media. J. Comput. Phys. 157 (2000), 44-88. Zbl 0960.74076 MR 1736884 
44. McCormack, M., Khachaturyan, A. G., \& Morris, J. W. Two-dimensional analysis of the evolution of coherent precipitates in elastic media. Acta Metall. 40 (1992), 325-336.

45. Miranville, A. Generalized Cahn-Hilliard equations based on a microforce balance. J. Appl. Math. 4 (2003), 165-185. Zbl 1031.35003 MR 1981620

46. Miranville, A. Generalizations of the Cahn-Hilliard equation based on a balance law for microforces. Gakuto Int. Ser. Math. Sci. Appl. 20 (2004), 189-211. Zbl 1068.35043 MR 2087470

47. Nikulin, Y., Stevenson, A. W., \& Hashizume, H. Model-independent determination of the strain distribution for a $\mathrm{Si}_{0.9} \mathrm{Ge}_{0.1} / \mathrm{Si}$ superlattice using x-ray diffractometry data. Phys. Rev. B $\mathbf{5 3}$ (1996), 82778282.

48. Nochetto, R. H. Finite element methods for parabolic free boundary problems. Adv. Numer. Anal. 1 (1991), 34-95. Zbl 0733.65089 MR 1138471

49. Novick-Cohen, A. On the viscous Cahn-Hilliard equation. In: Material Instabilities in Continuum Mechanics (Edinburgh, 1985-1986), Oxford Univ. Press (1988), 329-342. Zbl 0632.76119 MR 0970531

50. Nye, J. F. Physical Properties of Crystals. Clarendon Press, Oxford (1964).

51. Onsager, L. Reciprocal relations in irreversible processes I. Phys. Rev. 37 (1931), 405-426. Zbl 0001.09501

52. Onsager, L. Reciprocal relations in irreversible processes II. Phys. Rev. 38 (1931), 2265-2279. Zbl 0004.18303

53. ONUKI, A. Ginzburg-Landau approach to elastic effects in the phase separation of solids. J. Phys. Soc. Japan 58 (1989), 3065-3068.

54. PAWŁOW, I., \& ZAJĄZKOWSKI, W. M. Strong solvability of 3-D Cahn-Hilliard system in elastic solids. Math. Methods Appl. Sci. 31 (2008), 879-914. Zbl 1145.35004 MR 2418670

55. Svoboda, J., \& LuKÁs, P. Model of creep in $\langle 001\rangle$-oriented superalloy single crystals. Acta Mater. 46 (1998), 3421-3431.

56. Wasilewski, Z. R., Dion, M. M., Lockwood, D. J., Poole, P., Streater, R. W., \& SpringThorpe, A. J. Composition of AlGaAs. J. Appl. Phys. 81 (1997), 1683-1694.

57. Watson, S. Crystal growth, coarsening and the convective Cahn-Hilliard equation. In: Free Boundary Problems (Trento, 2002), Int. Ser. Numer. Math. 147, Birkhäuser (2004), 329-341. Zbl 1040.35078 MR 2044584 